\title{
Fluctuations in functional connectivity associated with interictal epileptiform discharges (IEDs) in intracranial EEG
}

\author{
Jennifer Stiso ${ }^{1,2}$, Lorenzo Caciagli ${ }^{2}$, Peter Hadar ${ }^{3}$, Kathryn A. \\ Davis $^{3}$, Timothy H. Lucas ${ }^{3}$, and Danielle S. Bassett ${ }^{2,4,5,6,7,8,9}$ \\ ${ }^{1}$ Neuroscience Graduate Group, Perelman School of Medicine, \\ University of Pennsylvania, Philadelphia, PA 19104, USA \\ ${ }^{2}$ Department of Bioengineering, School of Engineering and Applied Science, \\ University of Pennsylvania, Philadelphia, PA 19104, USA \\ ${ }^{3}$ Department of Neurology, Perelman School of Medicine, \\ University of Pennsylvania, Philadelphia, PA 19104, USA \\ ${ }^{4}$ Department of Electrical $\mathcal{G}$ Systems Engineering, School of Engineering $\mathcal{G}$ Applied Science, \\ University of Pennsylvania, Philadelphia, PA 19104, USA \\ ${ }^{5}$ Department of Psychiatry, Perelman School of Medicine, \\ University of Pennsylvania, Philadelphia, PA 19104, USA \\ ${ }^{6}$ Department of Physics 83 Astronomy, College of Arts 83 Sciences, \\ University of Pennsylvania, Philadelphia, PA 19104, USA \\ ${ }^{7}$ The Santa Fe Institute, Santa Fe, NM 87501, USA and \\ ${ }^{8}$ To whom correspondence should be addressed:dsb@seas.upenn.edu
}

\begin{abstract}
All epilepsies are defined by a propensity for recurrent seizures, characterized by hypersynchronous electrographic activity. Understanding this overarching property would be advanced by a thorough quantification of how the global synchrony of the epileptic brain responds to small perturbations that do not trigger seizures. Here, we leverage analysis of transient focal bursts of epileptiform activity, termed interictal epileptiform discharges (IEDs), to characterize this response. Specifically, we use a group of 145 participants implanted with intracranial EEG (iEEG) electrodes to quantify changes in 5 functional connectivity measures associated with three properties of IEDs: their presence, spread, and number. We perform this analysis in 5 frequency bands in order to contextualize our findings in relation to ongoing neural processes at different spatial and temporal scales. We find that, across frequency bands, both the presence and spread of IEDs tend to lead to independent increases of functional connectivity, but only in functional connectivity measures influenced by the amplitude, rather than the phase, of a signal. We find that these increases are not explained by simple subgroups of connections, such as the weakest connections in the brain, or only connections within the seizure onset zone. Evaluating patterns of similarity across different bands and measure combinations, we find that the presence of IEDs impacts high frequencies (gamma and high gamma) and low frequencies (theta, alpha, and beta) differently, although responses within each group are similar. Using grouped LASSO regression, we identify which individual-level features explain differences in functional connectivity changes associated with IEDs. While no single feature robustly explains observed differences, the most consistently included predictor across bands and measures is the anatomical locus of IEDs. Overall, this work provides compelling evidence for increases in global synchrony associated with IEDs, and delivers a thorough exploration of different functional connectivity measures, frequency bands, and IED properties. These observations show a disruption of several types of ongoing neural dynamics associated with IEDs. Additionally, we provide a starting point for future models of how small perturbations affect neural systems and how those systems support the hypersynchrony seen in epilepsy.
\end{abstract}

\section{INTRODUCTION}

Epilepsy is a heterogeneous neurological disease that is characterized by a predisposition towards recurrent seizures 1, 2]. While the different subtypes of epilepsy manifest in different types of seizures, levels of severity, and cognitive or somatic co-morbidities, the macroscale behavior of the brain exhibits a marked tendency towards hypersynchronous activity evident in seizures [1]. In order to better understand this property of hypersynchrony, it is useful to investigate how small perturbations affect endogenous patterns of neural synchrony. An interictal epileptiform discharge (IED) is a sub-seizure epileptic waveform that does not necessarily trigger the broad hy- persynchrony characteristic of seizure dynamics [3]. Nevertheless, IEDs often precede seizure activity and occur in spatially proximal regions 4 . IEDs have been considered the simplest phenomenon from which to study epilepsy 4, and have been shown to perturb ongoing cognitive processes [5]. Therefore, IEDs represent a promising candidate to better understand the synchrony of the epileptic brain through its response to small perturbations. Quantifying consistent changes in synchrony resulting from IEDs would represent an important step towards understanding the underlying properties of the epileptic brain that lead to seizures.

In EEG recordings, IEDs are single sharp, spike-like waveforms that result from a burst of firing in a small group of neurons 4]. IEDs have a quantifiable impact on 
neural dynamics and behavior. For example, these focal perturbations impact a battery of cognitive tasks, even when those IEDs occur outside the tissue that supports seizure formation, typically referred to as the seizure onset zone (SOZ) [5-7]. Additionally, IEDs are associated with spindle 8 and neural spiking $[9$ activity in regions distant from the source of the IED. These findings suggest that IEDs impact dynamics outside the population of cells producing the spike; however, a direct quantification of that impact in a large sample of source-level recordings has proven difficult, in part due to the scarcity of adequate data. Work using scalp EEG or functional MRI has indirectly addressed this question by trying to separate out the contribution of IEDs to group differences in synchrony observed between individuals with epilepsy and controls. Some work suggests that differences in intrinsic functional connectivity between individuals with and without epilepsy are largely due to IEDs, rather than to endogenous dynamics [10]. However, results vary across cohorts and methods for quantifying synchrony [11. Many studies have also detailed local or system-level changes in synchrony associated with IEDs, especially spatially near the onset of IEDs 12 14. These findings are corroborated by biophysical modeling studies showing a lack of global differences in functional connectivity associated with IEDs, but some local changes [15]. However, quantifying only local changes makes it difficult to connect these findings to the global synchrony seen in diverse seizure states.

A thorough investigation of the effect of IEDs on global synchrony is warranted to evaluate the response of an epileptic brain to perturbations. These observations could illuminate principles underlying the universal hypersynchrony seen across diverse seizure mechanisms. Here, we consider a thorough investigation to have two important properties: (1) it would comprehensively test different quantifications of global synchrony and (2) it would use a large sample to identify changes that are consistent across individuals. In EEG recordings, synchrony is quantified by an array of functional connectivity measures that identify statistical similarities between two signals. Additionally, EEG recordings provide information about synchrony in low frequency, high frequency, or broadband activity, each reflecting distinct neural processes. Low-frequency activity (theta, alpha, beta) in a single region is thought to reflect aligned fluctuations in the membrane potential of a large, local population of neurons. These fluctuations can modulate spiking activity to occur at the peaks of oscillatory rhythms [16]. Distant regions with similar lowfrequency activity, and therefore high functional connectivity, could be structurally connected regions with highamplitude oscillations where local spiking may or may not be modified [17. Some theories of cortical communication suggest a role for these spatially broad oscillations in top-down processing and attention[18, 19. Higher frequencies (especially high-gamma) in a single region are thought to correlate, though imperfectly [20], with local spiking activity 21, 22. Multiple regions showing similar high frequency activity, and therefore high functional connectivity, are thought to have similar local spiking patterns. Both gamma and high-gamma connectivity are theorized to support bottom-up processing [18, 19]. Identifying which, if any of these processes are changing during IEDs could help connect basic principles of hypersynchrony to underlying biological processes that generate activity.

Here, we use 145 individuals from the Restoring Active Memory (RAM) dataset 23, a publicly available dataset of individuals with epilepsy and intracranial EEG (iEEG) recordings to quantify changes in functional connectivity associated with IEDs that are not explained by changes in regional activity (Fig. 1A). We quantify connectivity based on phase (imaginary phase-locking value), amplitude (orthogonal amplitude envelope correlation) and amplitude-weighted phase (imaginary coherence) in 5 bands (3 low- and 2 high-frequency): theta, alpha, beta, gamma, and high gamma (Fig. 1 B). We also use the full broadband signal to investigate two measures that are not phase/amplitude separated: maximal cross-correlation and autoregressive fit. We use linear regression to quantify the amount that each of these metrics changes based on the presence of an IED, the number of IED sequences in a 1-second time window, and the average number of contacts containing an IED in each 1-second time window (Fig. 1 $\mathbf{C D}$ ). This information allows us to determine whether there are consistent changes to functional connectivity associated with IEDs, whether these effects impact local spiking connectivity or spatially distributed lower-frequency connectivity, and whether these effects selectively impact phase- or amplitude-based connectivity. After identifying increases in functional connectivity associated with IEDs, we ask whether these changes are driven by specific edges. We first hypothesize that increases might be driven by changes to the tails of the distribution that capture the weakest and strongest edges. Given that interictal connectivity is characterized by a globally disconnected [24], but strongly intraconnected 25] seizure onset zone, we further hypothesized that increases might be driven selectively by changes within the seizure onset zone. After testing these hypotheses, we explore patterns of similarity across measures and bands. We conclude by assessing possible sources of individual differences in the magnitude of IED effects. This work provides a thorough quantification of changes in global functional connectivity beyond changes in activity associated with different properties of IEDs, and identifies several candidate neural processes that are disrupted during these perturbations. These observations contextualize IEDs within the complex ongoing dynamics of the brain, and can be used to validate models that test principles underlying the hypersynchrony in epilepsy. 

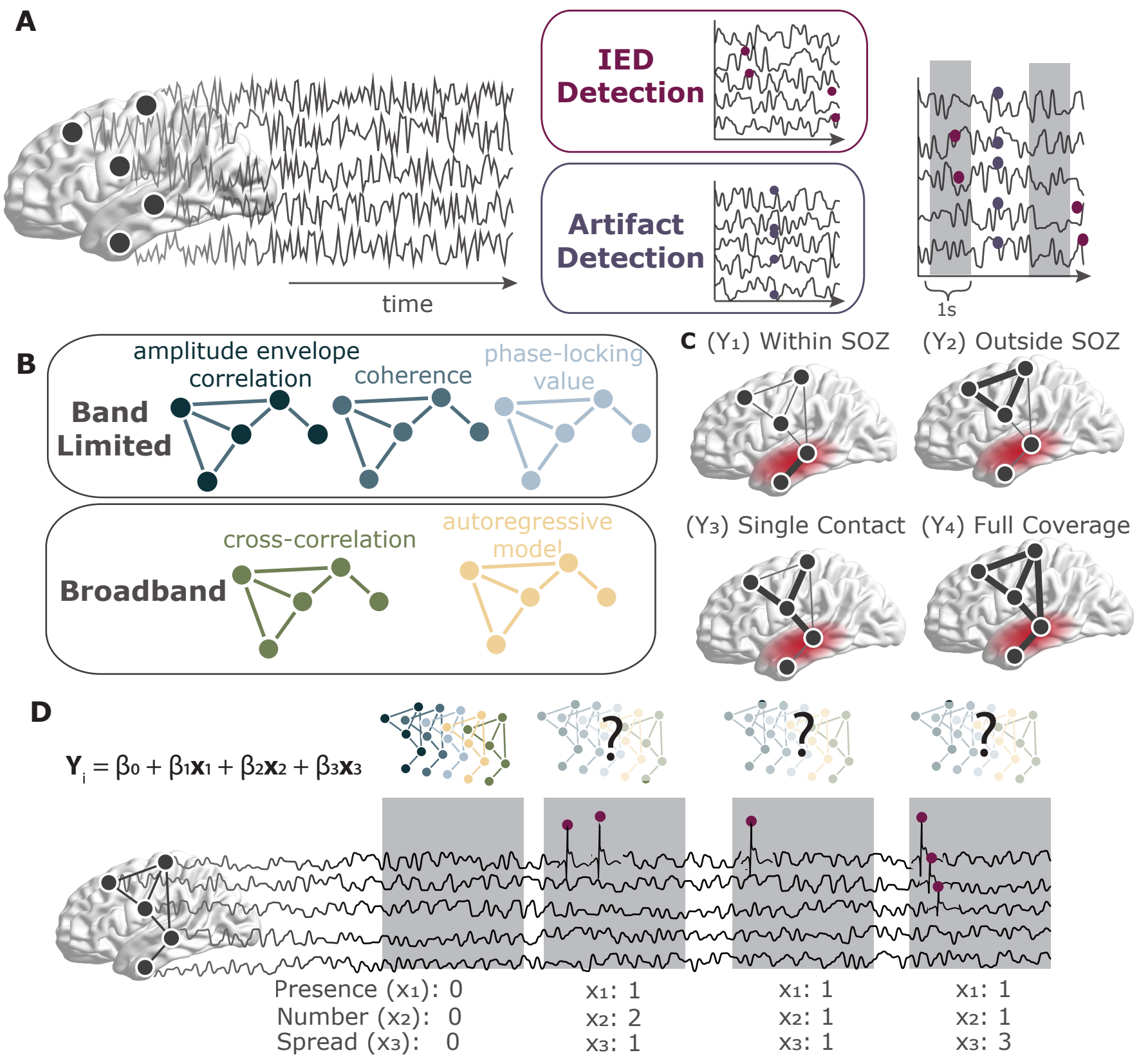

FIG. 1. Schematic of methods. (A) Data undergo automatic IED and artifact detection before being separated into 1-second windows. (B) Description of the different functional connectivity measures being tested. (C) Description of the different summaries of global functional connectivity being tested. Red region indicates the seizure onset zone. (D) Schematic of regression equation used to estimate effect sizes for 3 different properties of IEDs: their presence, number, and spread.

\section{METHODS}

\section{Participants (RAM Dataset)}

A publicly available dataset of 251 individuals undergoing intraoperative monitoring of their focal seizures was obtained from
http://memory.psych.upenn.edu/RAM. In each subject, monitoring was conducted with either grid electrocorticography electrodes, stereo electroencephalography electrodes, or both. Recordings were obtained while participants completed one of 5 different tasks testing either memory or free recall. Some participants underwent stimulation during these tasks. This data was 
collected as part of a multi-site, collaborative effort at the University of Pennsylvania, Columbia University, Dartmouth College, Emory University, Thomas Jefferson University, the Mayo Clinic, the National Institutes of Health, the University of Texas Southwestern, Medtronic Inc., and the Lawrence Livermore National Labs. A flow chart of preprocessing steps is shown in Fig. S1.

\section{Preprocessing Electrocorticography Data}

First, raw data from the RAM dataset was segmented into 5-second or longer task-free epochs from either before or after task completion. If no information was available regarding the timing of task events, or if this information was inconsistent, the recording session was not processed. Data were then downsampled to the lowest sampling rate used across recording sites $-500 \mathrm{~Hz}$ - using the resample () function in MATLAB. Electric line noise and its harmonics at 60,120 , and $180 \mathrm{~Hz}$ (all sites were located within the United States) were filtered out using a zero phase distortion 4th order stop-band Butterworth filter with a $1 \mathrm{~Hz}$ width. This filtering was implemented using the butter() and filtfilt() functions in MATLAB. For impulse and step responses of this filter, see Supplemental Figure S2.

We then sought to remove individual channels that exhibited excessive noise or had poor recording quality. The size of the dataset prevented us from visually inspecting each recording thoroughly; instead, we rejected channels using both the notes provided within the RAM dataset and using strict automated methods. After removing channels marked as low quality in the notes, we further removed electrodes that had either (1) a line length greater than three times the mean[5], (2) a $z$-scored kurtosis greater than 1.5 [26], or (3) a $z$-scored power-spectral density dissimilarity measure greater than 1.5 27. The dissimilarity measure was the average of one minus the Spearman's rank correlation of that signal with the signals of all other channels. These automated methods were chosen to remove channels with excessive high-frequency noise, electrode drift, and line noise, respectively.

Data were then demeaned and detrended. Detrending was used instead of a high-pass filter to avoid inducing filter artifacts 28. Channels were then grouped by grid or depth electrode, and common average referenced within each group. Following the common average reference, plots of raw data and power spectral densities were visually inspected by an expert researcher with 7 years of experience working with electrocorticography data (J.S.) to ensure that data traces were of acceptable quality.

\section{Automatic Interictal Epileptic Discharge (IED) Detection}

Automatic IED detection is still an open area of research, and there is as yet no consensus on best practices 29]. In clinical settings, epileptologists still manually mark EEG recordings for spikes and seizures when monitoring patients, although levels of agreement across different experts are not high [30. We chose to use a previously validated IED detector from Janca et al. 31 because it is sensitive, fast, and requires relatively little data per subject.

This Hilbert-based method dynamically models background activity and detects outliers from that background. Specifically, the algorithm first downsamples the data to $200 \mathrm{~Hz}$, and applies a 10-60 $\mathrm{Hz}$ bandpass filter. The envelope of the signal is then obtained by taking the square of this Hilbert-transformed signal. In 5-second windows with an overlap of 4 seconds, a threshold $k$ is calculated as the mode plus the median and that threshold is used to identify IEDs. The initial $k$ value is set to 3.65 , which was determined through cross-validation in prior work 31.

In order to remove false positives potentially caused by artifacts, we apply a spatial filter to identified IEDs. Specifically, we remove IEDs that are not present in a $50 \mathrm{~ms}$ window of IEDs in at least 3 other channels. The $50 \mathrm{~ms}$ window was taken from papers investigating the biophysical properties of chains of IEDs, which tended to last less than $50 \mathrm{~ms} 32$. Spikes detected within $80 \%$ of contacts within $2 \mathrm{~ms}$ were discarded. A subset of spikes were then randomly selected and validated by a board certified epileptologist (K.D.).

In order to quantify the spread and number of IEDs, we also determined where sequences of temporally proximal IEDs began and ended, using a previously validated algorithm 33, 34. IEDs occurring within $50 \mathrm{~ms}$ of the first IED in the sequence, or within $15 \mathrm{~ms}$ of the previous IED were considered part of the same sequence. Sequences were discarded if $50 \%$ of the spikes in the sequence occurred within $2 \mathrm{~ms}$ of each other.

\section{Alternate IED Detector}

To ensure that our results were not due to the specific IED detector used, we reproduced our key findings using an alternate detector. The alternate detector was the Delphos (Detector of ElectroPhysiological Oscillations and Spikes) detector 35. This detector was designed to detect and distinguish between both oscillations and IEDs in the time-frequency representation of the signal [35]. IEDs were detected by analyzing the time width and frequency spread of peaks above a given threshold. Here, we chose a threshold value of 50 because it identified a similar number of IEDs as our primary detector. Hence, we know that changes between detectors are not driven by a different number of IEDs, 
but by differences in the properties of the individual IEDs discovered.

\section{Transient Temporal Artifact Rejection}

Visual inspection of the data revealed two types of temporally transient artifacts that we sought to remove from further analyses. The first type was sharp channel drift. Here, most channels simultaneously jump to a higher voltage before slowly drifting back to their original level. These sharp transients can cause artifacts that mimic oscillations when filtered 28]. To automatically detect these sharp artifacts, we calculated the rate of change of the timeseries and looked for large outliers. An absolute threshold of 30,000 microvolts per sample was used, as it was determined to be well outside the normal range for several randomly selected datasets, while still capturing artifacts (Fig. S3A). All time points where at least half of all channels contained values greater than this threshold were removed from further analyses.

The second type of artifact we observed was the presence of large periods of flatlining across all channels. This flatlining could induce artificially large connectivity estimates as well as increased false positives in the IED detector. Since the detector dynamically calculates threshold values, periods of flat lining will lead to especially low thresholds and therefore many detected IEDs. Therefore, in addition to removing the flatlining periods themselves, we also removed 5 seconds of data following the artifact. To identify periods of flatlining, we searched for time points in the data with extremely small variance across all channels. We used a value of 300 microvolts because it was shown to be outside of the normal range but able to capture flatlining for several randomly selected subjects (Fig. S3B). All identified artifacts were visually inspected by an expert researcher with 7 years of experience working with electrocorticography data (J.S.) to ensure that identification was working as expected.

\section{Dataset Rejection}

Our last quality control effort consisted of removing from further analysis entire datasets that were especially noisy. Visual inspection of the data revealed three features indicative of low-quality data: (1) rough rather than smooth power spectral densities, (2) power spectral densities with many sources of line noise, and (3) many temporal artifacts. To identify datasets with uncharacteristic power spectra, we first calculated the average power spectral density across all channels using Welch's method in $500 \mathrm{~ms}$ windows (pwelch() in MATLAB). We then calculated the Spearman's rank correlation between the power spectral densities of all datasets. We removed the $10 \%$ of datasets with the lowest correlations.

To remove datasets with high levels of line noise, we started with the same power spectral densities calculated above, and sought to identify those that were not fit well with a smooth curve. We then fit a smooth spline function to each power spectral density using fit() in MATLAB with a smoothing parameter of $0.01 \mathrm{~Hz}$. Frequencies with notch filters and frequencies below $10 \mathrm{~Hz}$ were excluded from the fit, since they often deviated from the smooth curve and increased error rates. For each dataset, we then calculated the sum of squared errors between the smooth fit and the power spectral density. Power spectral densities with line noise at many frequencies would not be fit well by the smooth curve, and have higher errors. We then removed the $10 \%$ of datasets with the highest error.

Lastly, we rejected any dataset with greater than 1,000 time points containing temporal artifacts. Most of these datasets were also removed by one of the other methods. Lastly, we visually inspected the remaining data to confirm that the retained datasets looked clean. After performing these steps, we were left with data from 181 participants.

\section{Dynamic Functional Connectivity}

In this work, we seek to quantify how functional connectivity between brain regions changes in association with IEDs. To test for these changes, we first split all task-free data into 1 second non-overlapping windows. If a window contained an IED, that window was realigned to start 1 sample before the first IED in that window. Successive windows would be shifted later so that they did not overlap. Once windows were defined, data were prewhitened within each window using the Fieldtrip Toolbox for MATLAB 36 (ft_preprocessing() function with the derivative parameter). To systematically explore the changes in functional connectivity following IEDs, we calculated 5 different commonly used metrics. For band-limited measures in the theta $(\theta, 4-8 \mathrm{~Hz})$, alpha $(\alpha, 9-15 \mathrm{~Hz})$, beta $(\beta, 16-25 \mathrm{~Hz})$, and gamma $(\gamma$, $36-70 \mathrm{~Hz}$ ) bands, we wished to investigate amplitudebased, phase-based, and combined metrics (band definitions taken from Refs. [37, 38]). Therefore, we calculated orthogonal amplitude envelope correlations (amplitude), imaginary phase-locking value (phase), and imaginary multitaper coherence (combined). We also calculated the power in each band for each window, which we include as a covariate in later analyses. For broadband measures, we sought to characterize both undirected functional connectivity and directed effective connectivity. Therefore, we calculated the maximal cross-correlation, as well as a vector autoregressive model.

Orthogonal Amplitude Envelope Correlation: The amplitude envelope correlation (AEC) quantifies the ex- 
tent to which signals from two channels change amplitude synchronously. For each bandpass filtered timeseries $y_{n}(t)$ at channel $n$, the instantaneous amplitude is obtained from the analytic signal $z_{n}(t)$ where $z_{n}(t)=$ $y_{n}(t)+i y_{H n}(t)=\rho_{n}(t) e^{i \phi_{n}(t)}$ and $\phi_{n}(t)$ is the instantaneous phase. Correlations between these amplitude envelopes are highly susceptible to artificial correlations due to volume conduction 39,40 . In electrophysiological signals specifically, similarities between channels at location A and B could be caused by the signal from a third source $\mathrm{C}$ that spreads through the tissue and cerebrospinal fluid and is picked up by both channels, despite region $\mathrm{C}$ having no functional relationship to regions $\mathrm{A}$ and $\mathrm{B}$. This process is called volume conduction, and while it is a much bigger problem for sensor-level recordings (EEG and magnetoencephalography (MEG)), it can still have effects on iEEG data. Therefore, we take only the orthogonal components of each signal before calculating their correlation.

Here, we account for volume conduction using the method from Nolte et al. 40. We chose this method as opposed to the method from Hipp et al. [39, because it uses a global normalization constant rather than one fit to each time point and is therefore much faster. Assuming Gaussian distributed data, which is a reasonable but not perfect fit to short segments of electrophysiological recordings, we can obtain the portion of the analytic signal $z_{m}$ that is orthogonal to $z_{n}$ by subtracting $z_{n}$ multiplied by the real part of the coherency spectrum between the two channels. Specifically, we first normalize the analytic signal from channels $n$ and $m$ such that $<\left|z_{n}\right|^{2}>=<\left|z_{m}\right|^{2}>=1$. Here, the expected value is taken over time points. We then calculate coherency $c$ as $c=\frac{<z_{n} z_{m} *>}{\left(\left(z_{n} z_{n} *\right)\left(z_{m} z_{m} *\right)^{1 / 2}\right.}$ where $*$ refers to the complex conjugate. Lastly, we calculate the correlation between $z_{n}$ and $z_{m}-\operatorname{real}(c) z_{n}$. The resulting value ranges from -1 to 1 , with 1 indicating perfectly correlated signals, and -1 indicating perfectly anti-correlated signals. The absolute value of the orthogonal amplitude envelope correlation was taken before averaging across all pairwise estimates, or edges, in the functional network.

\section{Bandpass Filter - Imaginary Phase-Locking Value (PLV)}

The phase-locking value quantifies the temporal consistency of the phase offset between two channels regardless of the signal amplitude. The imaginary phase locking value removes 0 -phase lag contributions to this value, which could arise from volume conduction. Using the bandpassed signal, $y_{n}(t)$, obtained with the parameters listed above, we calculate the imaginary phase locking value as $i P L V_{i, j}=\frac{1}{T} \operatorname{imag}\left(e^{-i \phi_{i}(t)-\phi_{j}(t)}\right)$. Here, $\phi_{i}$ is the instantaneous phase in channel $i$ for a given frequency band. This process was implemented using custom code in MATLAB, taken from Ref. 41]. Instantaneous phase requires a narrow frequency range in order to be biologically interpretable; therefore, we do not compute this measure on the high gamma band[42]. This measure ranges between 0 and 1 , where 1 indicated consistent and small phase offsets, and 0 indicates inconsistent or large phase offsets.

\section{Multitaper Fourier Transform - Imaginary Coherence 8 Power}

Multitaper Fourier Transform: Multitaper fast Fourier transforms (FFTs) use multiple tapers in order to better control spectral leakage at high frequencies 43 . They can be used to obtain the cross-spectral density, $S_{i, j}$, between any two channels $i$ and $j$ as well as the power spectral density $S_{i i}$ for channel $i$. The multitaper FFT was computed on 30 logarithmically spaced frequencies between 4 and $150 \mathrm{~Hz}$ using discrete prolate spheroidal sequences (DPSS) tapers with $4 \mathrm{~Hz}$ smoothing. Recording windows were zero-padded to the maximum period length for a given frequency. This calculation was implemented in MATLAB with the Fieldtrip toolbox 36 .

Imaginary Coherence: Coherence quantifies the consistency of phase offsets between two channels, weighted by their signal amplitude. Imaginary coherence ignores the contribution of 0-phase lag signals to this value, which could arise from volume conduction. Mathematically, the complex valued coherence $c$ between two signals is $c=\frac{S_{i, j}}{\sqrt{S_{i i}^{2} S_{j j}^{2}}}$. The imaginary coherence $C$ is then taken as the absolute value of the imaginary component of coherence, $|\operatorname{imag}(c)|$. Imaginary coherence ranges from 0 to 1 . Imaginary coherence values were averaged across all frequencies within a band.

Power: We wished to quantify changes to connectivity above what would also be explained by changes in activity. Here, we quantify activity as spectral power. Spectral power for each band and each channel was calculated by taking the logarithm (base 10) of all frequencies that fell within a given band.

\section{Low-Pass Filter - Cross-Correlation}

Low Pass Filter: For broadband measures, we first removed sources of high-frequency noise using a low pass filter. Data were low pass filtered at $200 \mathrm{~Hz}$ using a 0phase lag 4th order Butterworth filter. This filter was implemented using the MATLAB package Fieldtrip.

Cross Correlation: The cross-correlation is the maximal correlation between two signals that can be achieved across shifts of one signal relative to the other. The correlation coefficient was normalized such that the autocorrelation at zero lag was equal to one. The cross-correlation was implemented with the MATLAB function $x \operatorname{corr}()$. The resulting values range between 0 and 1 . 
Low-Pass Filter - Effective Connectivity from Autoregressive (AR) Models

Autoregressive (AR) Models: All the measures described above are undirected functional connectivity measures that will always provide the same estimates of the relation between node $i$ and $j$ as of the relation between node $j$ and node $i$. We also wished to consider a directed measure of connectivity, in which the influence of node $i$ on node $j$ could be different from the influence of node $j$ on node $i$. To accomplish this goal, we calculated a measure of directed connectivity from the weights of a first-order autoregressive model fit to the data. The weights were obtained by solving for $(A)$ in the equation $\mathbf{x}(\mathbf{t})=\mathbf{A} \mathbf{x}(t-1)+\epsilon(t)$, where $\mathbf{x}$ is the timeseries data, and $\epsilon$ is an error term. Fits were calculated using the arfit package in MATLAB 44]. Connectivity values from AR models range from -1 to 1 , so the absolute value was taken before summarizing. AR models assume a linear relationship between signals and assume the data are stationary. For short time windows (here, 1 second), this assumption is reasonable and has been made in previous work 45 .

\section{Estimating Effects of IEDs on Functional Connectivity}

The overarching goal of this study is to quantify the effect of IEDs on each of the functional connectivity metrics defined above. We also wished to be able to answer the three specific questions: (1) Are changes in connectivity driven by shifts in the weakest or strongest connections? (2) Are changes larger within than outside the seizure onset zone? (3) Do effects of IEDs vary reliably across regions or individuals? To answer these questions, we calculated IED effects on 5 different summary measures of the distributions of connections. These measures included (1) the average connectivity across all contacts (full coverage), (2) the skew of connectivity across all contacts, (3) the strength of connectivity inside the seizure onset zone, (4) the strength of connectivity outside the seizure onset zone, and (5) the strength of connectivity in each individual contact (Fig. S1C). In supplemental analyses, we also report the changes in connectivity in two additional summary measures: (1-2) between all contacts inside and outside the irritative zone, (3-4) the strength between all grid and depth contacts separately, and (5-6) the strength between grey and white matter portions of depth contacts.

We next quantify the specific features of each IED sequence that we hypothesized would impact functional connectivity uniformly. The first feature is the presence of an IED in a window, regardless of the properties of that IED. The second is the number of sequences within a window. A sequence is defined as all IEDs occurring within $50 \mathrm{~ms}$ of the first IED, or within $15 \mathrm{~ms}$ of the last
IED in that timeframe. If the leader channel had multiple spikes in this sequence, the initial sequence was split into multiple sequences. The third is the average spread across each sequence within the window (Fig. S1D).

For each dependent variable, we calculated the coefficient of each IED predictor in a permutation-based linear model that contained power and recording session as nuisance covariates. If two or more of the three covariates of interest were perfectly colinear, one of them was removed from the model. Typically, colinearity occurred when one or more predictors did not vary. For example if all windows contained only 1 IED, the presence and number of IED predictors would be colinear. The results presented in the main text are obtained from a subset of 145 subjects that had coefficient values for all three predictors. Outliers, defined as values 3 standard deviations smaller or larger than the mean distribution for each band and measure combination, were removed from further analyses. Permutation-based models were used because both the spike spread and the number of spikes are highly skewed, non-normal variables that could lead to artificially large estimations in parametric models. Additionally, permutation-based models more effectively down-weight outliers in the distribution.

\section{Individual-Level Variables}

We investigated the relative efficacy of 12 variables in explaining changes in functional connectivity associated with IEDs: age, sex, handedness, race, locus of IEDs, hemisphere of IEDs, etiology, the presence of a lesion, the age of seizure onset, the subject's average task performance, the institution of treatment, and the type of contacts (grid or depth electrodes). Of these variables, sex, race, institution, age, type of coverage, and handedness were included as they were recorded in the public data release. If subjects had missing values in any of these demographic variables, they were filled in with the most common demographic for categorical variables, or the mean demographic for continuous variables. Other variables used in later analyses required processing before including them in the model or were not included in the public data release. These will be described below.

\section{Clinical Variables}

We received additional clinical data that were not part of the public release from the creators of the original study. These data included: age at seizure onset, presence of a lesion, and the underlying etiology. The underlying etiology was categorized as follows: (1) traumatic brain injury, (2) infection, (3) neurocutaneous syndrome, (4) neoplasia, (5) stroke, (6) malformation of cortical development, (7) medial temporal sclerosis, (8) hypoxemic ischemic encephalopathy, (9) other identified etiologies 
in a different category, and (10) unknown etiology. The variable with missing values for the most people was etiology, which was missing in $79.3 \%$ of participants. Here, we note that these missing values are different from the unknown etiology, which notes that clinicians were unable to determine etiology, rather than that etiology was not recorded. Individuals with missing values were not included in our final analysis.

\section{Contact Variables}

In various analyses, we have used information about the locus, hemisphere, and tissue type (grey or white matter) for each contact. The locus and hemisphere of IEDs were obtained from information provided in the public data release. Coordinates provided in Montreal Neurological Institute (MNI) space were registered to the Schaefer parcellation [46] of 7 cognitive systems 47]. It is standard practice to register atlases to a subject-specific space, rather than registering subject coordinates to a common space. To confirm the accuracy of system assignments, once each contact had been assigned a system, we manually checked that the physician-assigned regions (the 'region' field in the data release) of each contact matched their given system. Participants with missing MNI coordinates were excluded from these analyses $(73.8 \%)$. White and grey matter labels for each contact were obtained from the Neuromorphometrics cortical parcellation protocol 48 given in the 'wb' field of the data release.

\section{Task Performance}

The last included variable was each participant's average task performance on a subset of 5 tasks. Two tasks were free recall tasks 23, 49, 1 was associative learning, and 2 were spatial navigation tasks 49] (see RAM documentation for further details). For all tasks, performance ranges from 0 to 1 ; however, a value of 1 in free recall tasks indicated good performance, while a value of 1 in navigation tasks indicated poor performance. We averaged each participant's reverse score on all free recall tasks with $(1-$ navigation $)$. Most subjects $(97.2 \%)$ completed at least 1 task, but those who did not were removed from these analyses.

\section{Group LASSO}

We used group LASSO regression to assess the relative importance of each variable. Group LASSO applies a penalty to groups of variables and will regularize coefficients of uninformative groups to 0 . Here, all levels of a given categorical variable were grouped together. This way, all levels of the etiology variable, for example, would be regularized together, rather than regularizing individual levels. Group LASSO was implemented using the gglasso package in $\mathrm{R}$ (https://github.com/emeryyi/gglasso).

\section{Statistical Analyses}

Here, we set out to complete an exploratory analysis of the changes to functional connectivity associated with IEDs. We used a strict family-wise Bonferroni correction in which each band/measure combination was treated as a different hypothesis. This procedure allowed us to have more confidence in the results we report, but its stringency may contribute to false negatives.

Before any significance testing, subjects with changes to functional connectivity greater than 3 standard deviations above the mean were removed. Quantile-quantile plots were then used to check whether distributions were normal. Since distributions appeared to deviate from a normal distribution, one sample permutation tests assessed whether distribution means were significantly different from 0 (Figs. 2, 3). Statistics are reported with the test statistic, the number of observations, and the $p$-value. Similarity was assessed with Spearman's correlations (Figs. 45 to mitigate the influence of outliers.

Differences within versus between groups in similarity matrices were assessed with Pearson's correlations between the lower diagonal of matrix entries, and a binary mask specific to each hypothesis. Pearson's correlations assume independent observations, which is not the case for the entries in the similarity matrices used for Figs. 4.5. Using tests intended for independent observation on clustered data can result in $p$-values that are artificially small due to the artificially high sample size [50. However, in these analyses, it is not the assessment of the significance of correlations that interests us, but rather the relative similarity values that are found with different masks. Accordingly, we include Pearson's $r$ as a simple and interpretable estimate of similarity. To assess the significance of these measurements, we tested correlations calculated from empirical similarity matrices against correlations calculated from 100 permuted null models. In each null model, a random mapping of true band-measure pairings to permuted band-measure pairing was given to all participants.

\section{Data and Code}

Code is available at github.com/jastiso/interictal_fc. Data is publicly available at http://memory.psych.upenn.edu/RAM. 


\section{RESULTS}

We sought to characterize the changes in global functional connectivity associated with a simple form of epileptic activity - an IED - in individuals with medically refractory epilepsy. Accordingly, we used a large sample of 145 participants in the RAM dataset. We select a comprehensive sample of functional connectivity measures common to iEEG analysis. Specifically, we calculate 3 measures of band-limited functional connectivity and 2 measures of broadband functional connectivity in $1 \mathrm{~s}$ windows. Next, we quantified the effect of 3 different properties of IEDs on each bandmeasure combination using a permutation-based linear model including the presence of an IED, the number of IEDs in a window, and the average spread of every IED in the window (Fig. 1D). In order to identify drivers of spatial distributions of observed effects, we calculate the effects of IEDs on the total strength across all contacts, the skew of edges across all contacts, the strength of only those contacts within (or outside) the seizure onset zone, and in each contact individually (Fig. 1 C).

\section{Quantifying the impact of IEDs on functional connectivity}

We first asked which band-measure combinations would show consistent changes in the strength of functional connections for each of the 3 IED predictors. We find different patterns of responses across predictors. The presence of IEDs increases functional connectivity, but largely in the orthogonal amplitude envelope correlations (Fig. $\mathbf{2} \mathbf{A}$; one-sample permutation test, Bonferroni-corrected $p$-values $(n=16) d_{\theta, A E C}(141)=$ $0.25, p=0.013, d_{\beta, A E C}(143)=0.45, p<1.0 \times 10^{-4}$, $d_{\beta, i C o h}(140)=0.19, p<1.0 \times 10^{-4}, d_{\gamma, A E C}(142)=0.22$, $p<1.0 \times 10^{-4}, d_{\gamma, i C o h}(141)=0.60, p<1.0 \times 10^{-4}$, $\left.d_{\gamma+, A E C}(142)=0.49, p<1.0 \times 10^{-4}\right)$. The number of IEDs in a window does not consistently affect functional connectivity. The only exceptions were the orthogonal amplitude envelope correlation in the beta band and high gamma band (Fig. 2 $\mathbf{2 B}$; one-sample permutation test, Bonferroni-corrected $p$-values $(n=16) d_{\gamma+, i C o h}(142)=$ $0.14, p<1.0 \times 10^{-4}, d_{\text {broadband,xcorr }}(143)=1.18$, $\left.p<1.0 \times 10^{-4}\right)$. The spread of IEDs within a window largely increases both amplitude-based (orthogonal amplitude envelope correlation) and amplitude weighted phase-based (imaginary coherence) measures (Fig. $2 \mathbf{2}$; one-sample permutation test, Bonferroni-corrected $p$ values $(n=16) d_{\theta, A E C}(142)=0.065, p=6.4 \times 10^{-3}$, $d_{\theta, i C o h}(142)=0.056, p=3.2 \times 10^{-3}, d_{\theta, i P L V}(143)=$ $-0.076, p<1.0 \times 10^{-4}, d_{\alpha, A E C}(142)=0.079, p<$ $1.0 \times 10^{-4}, d_{\alpha, i C o h}(142)=0.064, p<1.0 \times 10^{-4}$, $d_{\beta, A E C}(140)=0.13, p<1.0 \times 10^{-4}, d_{\beta, i C o h}(141)=$ $0.076, p<1.0 \times 10^{-4}, d_{\gamma, A E C}(142)=0.22, p<1.0 \times 10^{-4}$, $d_{\gamma, i C o h}(141)=0.061, p<1.0 \times 10^{-4}, d_{\gamma+, A E C}(141)=$
$0.11, p<1.0 \times 10^{-4}, d_{\gamma+, i C o h}(142)=0.033 \mathrm{i} p=$ $\left.6.4 \times 10^{-3}, d_{\text {broadband, } x \text { corr }}(142)=0.038, p=0.035\right)$. The predictor for the spread of IEDs also shows changes to functional connectivity that are an order of magnitude smaller than the other two predictors. Our results, based on a large sample $(n=145)$ of source-level data, demonstrate consistent increases in global functional connectivity as a result of the presence and spread of IEDs.

To ensure that results are driven by IEDs themselves, and not by artifacts due to our specific IED detection algorithm, we repeated the above analysis with a second IED detector. This detector used spectrotemporal features rather than the magnitude of the bandpassed signal to identify IEDs. With this alternative approach, we found significant effects for all but 4 of the measures listed above: the high gamma imaginary coherence (number); broadband cross-correlation (number); theta imaginary phase-locking value (spread); and broadband cross-correlation (spread) (Fig. 2 significance indicated with dark red asterisks). Using this algorithm, we also identified 4 additional measures that had a mean significantly different from 0 . These measures were the alpha orthogonal amplitude envelope correlation (presence), gamma imaginary phase-locking value (presence), gamma orthogonal envelope correlation (number), and the gamma phase-locking value (spread). This observation indicated that while the selection of IED detector can influence the quantification of neurophysiological changes associated with IEDs, most increases are consistent across detectors.

\section{Quantification in subsets of connections}

We next asked if these global effects were driven largely by smaller parts of the network. Because we found no reproducible large changes to functional connectivity for the number-of-IEDs predictor, we limited this analysis to the presence and spread of IEDs (for the number of IEDs, see Fig. S4). We hypothesized that increases in global connectivity were driven by a strengthening of the weakest connections, possibly connecting the seizure onset zone to the rest of the brain, or by a strengthening of already strong, isolated connections within the seizure onset zone. We broke these hypotheses first into two questions: (1) could increases be driven by the weakest edges? And (2) could increases be driven by edges in the seizure onset zone? To address our first question, we repeated the above analysis with the skew, rather than the strength of edges. Since edge distributions are heavy tailed (see Fig. S5), increases in the skew are consistent with a strengthening of the weakest edges in the distribution. We observe far fewer significant changes to the skew of connections than to the strength, and we note that these changes tend to be negative. Significant changes were seen in gamma orthogonal amplitude envelope correlation (presence), broadband cross-correlation (num- 
bioRxiv preprint doi: https://doi.org/10.1101/2021.05.14.444176; this version posted May 17, 2021. The copyright holder for this preprint (which was not certified by peer review) is the author/funder, who has granted bioRxiv a license to display the preprint in perpetuity. It is made

A
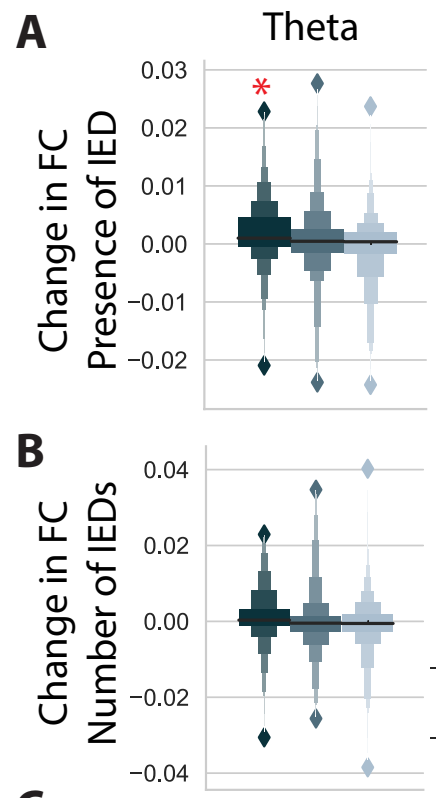

C

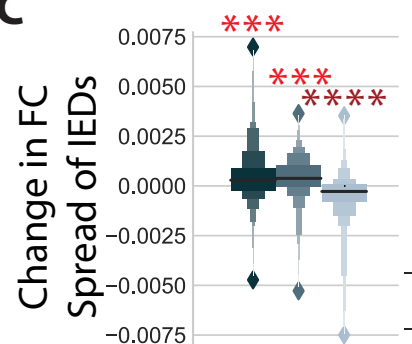

$-0.0075$
Alpha
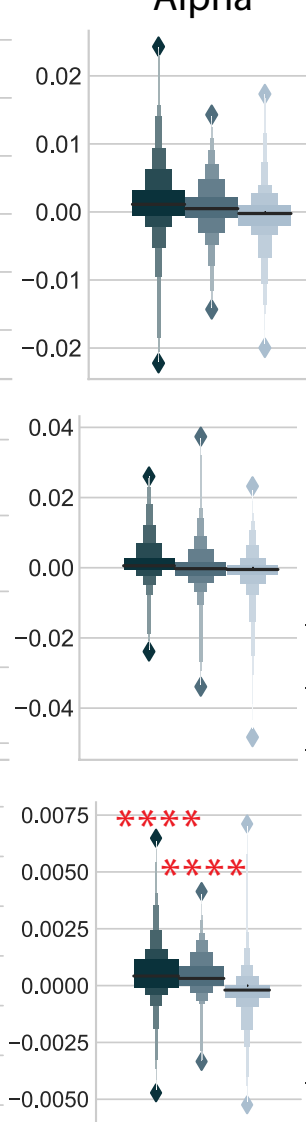

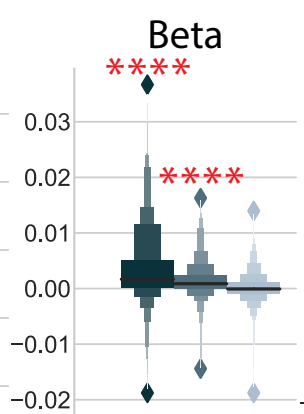

Beta
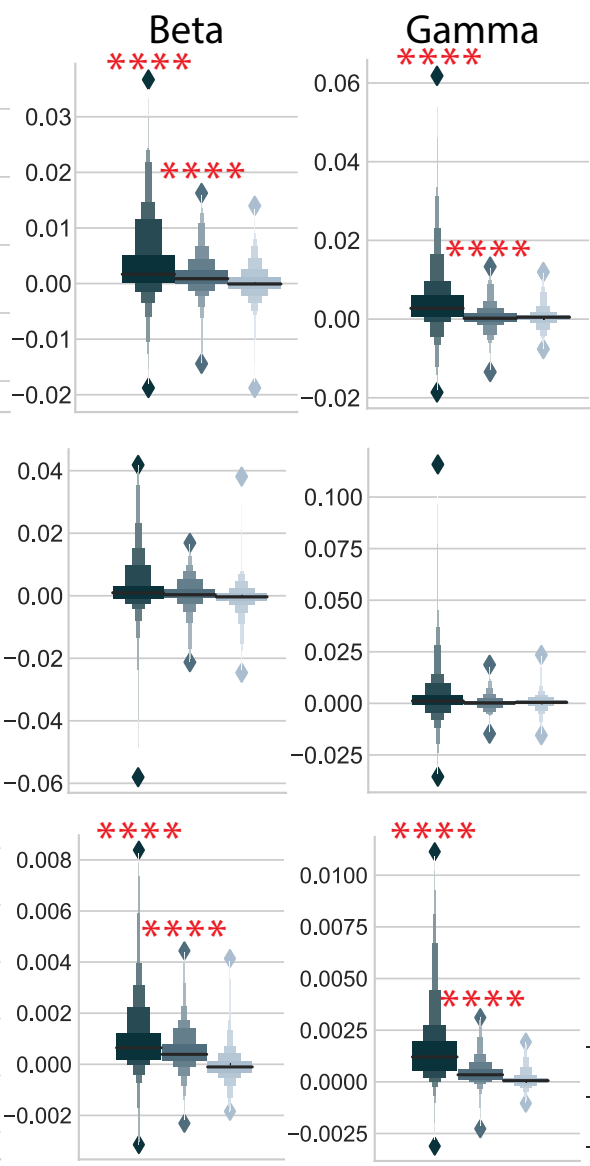
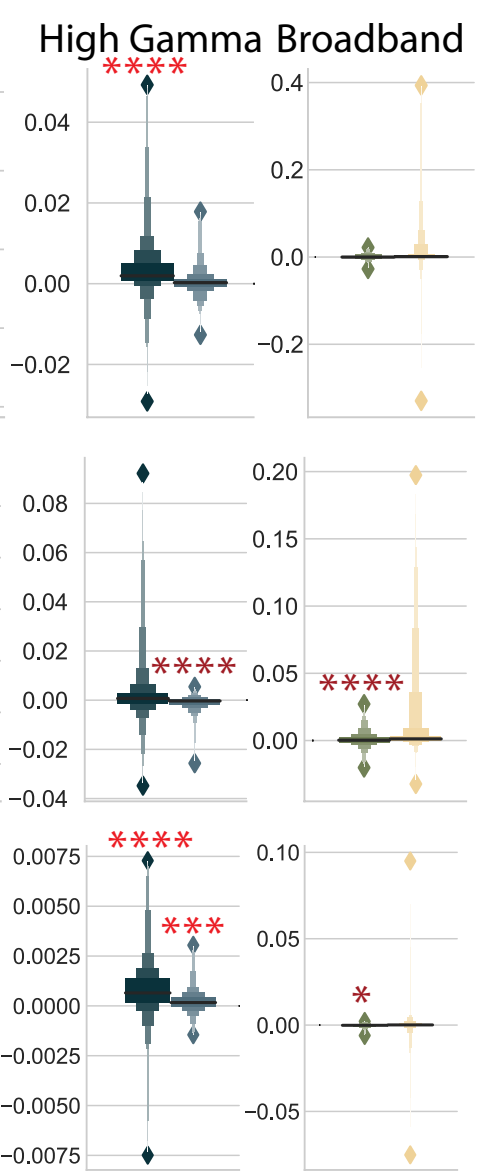

$\square$ Orthogonal AEC $\square$ Imag. Coherence

JImag. PLV $\square$ Cross-correlation

AR Model

FIG. 2. Changes in Functional Connectivity Associated with IEDs. (A) Magnitude of the change in functional connectivity (FC) for the presence of an IED obtained from permutation based regression including all IED predictors, power in a given band, and the recording session. Columns indicate different frequency bands, and colors indicate different measures. Bright red asterisks indicate significant distributions after multiple comparisons correction that could also be reproduced with a different spike detector. Dark red asterisks indicate effects that were not reproducible with another IED detector. ${ }^{*}=p<0.05$, ${ }^{* *}=p<0.01,{ }^{* * *}=p<0.001,{ }^{* * * *}=p<0.0001$. (B,C) Same as in panel $(A)$, but for the coefficients associated with the number of IEDs and the spread of IEDs. Here, AEC stands for amplitude envelope correlation, imag. for imaginary, PLV for phase locking value, and AR for autoregressive model.

ber), theta and alpha imaginary coherence (spread), and beta and high gamma orthogonal amplitude envelope correlation (spread) (Fig. $\mathbf{3} \mathbf{A}$; one-sample permutation test, Bonferroni-corrected $p$-values $(n=16) d_{\gamma, A E C}(140)=$ $-2.26, p=3.2 \times 10^{-3}, d_{\text {broadband,xcorr }}(144)=-12.43$, $p=0.026, d_{\theta, i C o h}(142)=-0.39, p=6.4 \times 10^{-3}$, $d_{\alpha, i C o h}(143)=-0.44, p=0.019, d_{\beta, A E C}(141)=0.37$, $\left.p=3.2 \times 10^{-3}, d_{\gamma+, A E C}(140),=0.60, p<1.0 \times 10^{-4}\right)$. These findings indicate that most changes observed above are not driven by a strengthening of weak connections.

We address our second question by subtracting coefficients for the effect of IEDs on connections inside the clinically defined seizure onset zone (SOZ) from connections outside the SOZ, for participants where this data was available $(n=104)$. Large positive numbers would indicate greater changes inside the SOZ. We find that, after correction for multiple comparisons, only the changes in connectivity resulting from the spread of IEDs were larger in the seizure onset zone compared to the rest of the brain (Fig. $\mathbf{3} \mathbf{B})$. While these differences are seen in all but the theta band, they are most pronounced in the connectivity of the high gamma band (Fig. $\mathbf{3} \mathbf{B}$; one-sample permutation test, Bonferroni-corrected $p$ values $(n=16) d_{\alpha, i C o h}(102)=0.05, p=3.2 \times 10^{-3}$, $d_{\beta, A E C}(101)=0.07, p=3.2 \times 10^{-3}, d_{\beta, i C o h}(102)=$ $0.043, p=0.019, d_{\gamma, A E C}(101)=0.079, p=0.032$, $d_{\gamma, i C o h}(101)=0.033, p<1.0 \times 10^{-4}, d_{\gamma+, A E C}(100)=$ $0.083, p=3.2 \times 10^{-3}, d_{\gamma+, i C o h}(99)=0.030, p=$ $0.02, d_{\text {broadband, } A R}(104)=0.063, p=6.4 \times 10^{-3}$, $\left.d_{\text {broadband,xcorr }}(101)=0.066, p=0.030\right)$. Some mea- 
sures, indicated with a grey asterisk in Fig. 3 , showed significant uncorrected differences, and also suggested larger effects inside the seizure onset zone. To determine whether the changes in global functional connectivity associated with the spread of IEDs was driven by edges within the SOZ, we tested whether edges between contacts outside the SOZ showed changes in functional connectivity that were statistically different from 0 . Only one measure of the original 12 significant measures, the high gamma imaginary coherence, was no longer significant when only considering edges outside the SOZ. Overall, we find that only changes associated with the spread, and not the presence, of IEDs are increased within the seizure onset zone. While changes are larger within the $\mathrm{SOZ}$, edges outside the SOZ still show positive changes associated with the spread of IEDs.

We then tested our third hypothesis: that changes would be driven by the larger irritative zone (IZ) rather than the seizure onset zone (SOZ). Clinically, the IZ is defined as the portion of tissue generating IEDs. Changes limited to the IZ could be due to biological disruptions in ongoing interactions, or to spurious changes in statistical similarity associated with filtering artifacts arising from the spikes themselves. We repeated our analysis in participants with clinical markings of which channels belonged to the IZ $(n=101)$. We then calculated differences in coefficients between contacts in the IZ and contacts outside of the IZ. We find 8 significant differences after multiple comparisons correction for the spread of IEDs spanning all bands and measures (Fig. S6; one sample permutation test, Bonferroni-corrected $p$-values $(n=16) d_{\theta, i \text { Coh }}(99)=0.04, p=0.04, d_{\alpha, i C o h}(98)=$ $0.03, p=0.04, d_{\gamma, A E C}(96)=0.08, p<1.0 \times 10^{-4}$, $d_{\gamma, i \text { Coh }}(97)=0.03, p=6.4 \times 10^{-3}, d_{\gamma+, i C o h}=0.03$, $\left.p=1.0 \times 10^{-4}\right)$. We further tested whether only connections between channels without IEDs showed significant changes associated with IEDs in bands and measures that showed both significant increases in global connectivity associated with IEDs (Fig. 2) and significant differences between contacts with and without IEDs (one sample permutation test, Bonferroni-corrected $p$-values $(n=16)$ $d_{\theta, i \text { Coh }}(98)=0.045, p=0.013, d_{\alpha, i C o h}(99)=0.035$, $p<1.0 \times 10^{4}, d_{\beta, A E C}(97)=0.076, p<1.0 \times 10^{-4}$, $d_{\gamma, A E C}(97)=0.12, p<1.0 \times 10^{-4}, d_{\gamma, i C o h}(98)=0.039$, $p<1.0 \times 10^{-4}, d_{\gamma+, A E C}(99)=0.073, p=6.4 \times 10^{-3}$, $d_{\gamma+, i C o h}=0.037, p<1.0 \times 10^{-4}, d_{\text {broadband,xcorr }}(100)=$ $\left.0.071, p=6.4 \times 10^{-3}\right)$. We found that all but three of the original twelve measures remained significant after only considering connections between contacts outside the IZ. The three measures were the theta orthogonal amplitude envelope correlation, alpha orthogonal amplitude envelope correlation, and high gamma imaginary coherence. Overall, this finding suggests that the changes to functional connectivity associated with the spread of IEDs are larger in the clinically defined IZ, and sometimes limited to those regions. However changes associated with the presence and number of IEDs are not limited to any of the edge subsets we tested.
Given that the changes in functional connectivity associated with the presence of IEDs had a similar magnitude within and outside of pathological tissue, we hypothesized that changes might be driven by edges that sample tissue differently, or that are located in different types of tissue. In particular, we hypothesized that changes in functional connectivity might be driven by connections amongst grid contacts versus depth contacts, or within grey versus white matter on depth contacts. For all individuals who had a combination of grid and depth coverage $(n=35)$, we assessed whether functional connectivity changes were greater for edges within contacts in the grid versus edges within depth contacts. Due to the smaller sample size, we only tested this effect in functional connectivity band and measure combinations that showed significant changes in functional connectivity (the orthogonal amplitude envelope correlation and imaginary coherence for all bands). We find no significant differences between edges within grid and depth coverage, either before or after multiple comparisons corrections (Fig. S7). We then repeated this process for individuals who had depth contacts marked as grey and white matter $(n=$ 104). We found no significant differences between white and grey matter after multiple comparisons corrections, although some measures were significant before correction (Fig. S7).

\section{Relationship between IED predictors and changes to connectivity within frequency bands and connectivity measures}

Earlier, we explored a large space of IED predictors and their effects on different frequency bands and connectivity measures. In order to distill these many findings into broader, unifying principles regarding the behavior of neural systems during simple epileptiform events, it would be useful to determine whether there are consistent associations between different predictors, frequency bands, or measures. To identify these associations, we calculate similarity with Spearman's correlations of participants' coefficients between IED predictors and bandmeasure combinations.

We first computed pairwise correlations between the distribution of participants' coefficients for each of the IED predictors, in each band-measure combination. We find that relationships are largely consistent across bands, and we therefore show results from each measure averaged across bands (Fig. 44 $\mathbf{A}$ ). We find that the strongest effect is a significant positive correlation between the magnitude of changes associated with the number of IEDs and the presence of an IED (Fig. $4 \mathbf{A}$; Spearman's correlation, Bonferroni-corrected $p$-values $(n$ $=16)$ mean $r_{A E C}=0.66+/-0.061$, mean $r_{i C o h}=0.57+$ $/-0.14$, mean $r_{i P L V}=0.60+/-0.070, r_{x c o r r}=0.57$, $r_{A R}=0.70$, all $\left.p<5.14 \times 10^{-5}\right)$. The correlations between the connectivity changes associated with the pres- 
bioRxiv preprint doi: https://doi.org/10.1101/2021.05.14.444176; this version posted May 17, 2021. The copyright holder for this preprint (which was not certified by peer review) is the author/funder, who has granted bioRxiv a license to display the preprint in perpetuity. It is made available under aCC-BY-NC-ND 4.0 International license.
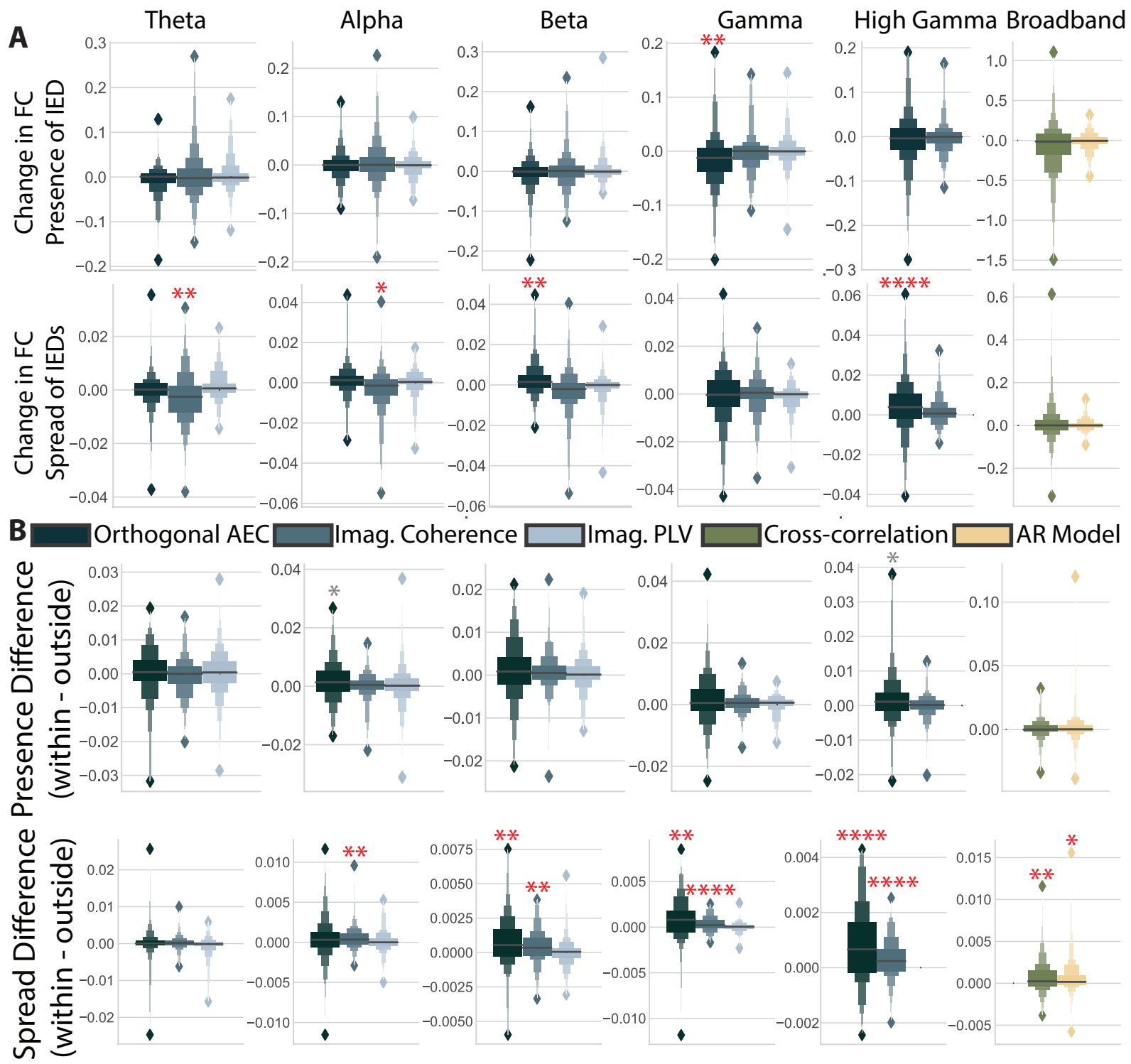

FIG. 3. Changes to specific connections. (A) The coefficient associated with each predictor for explaining changes to the skew of the distribution of functional connectivity. ${ }^{*}=p<0.05,{ }^{* *}=p<0.01,{ }^{* * *}=p<0.001,{ }^{* * * *}=p<0.0001$. Grey asterisks indicate significant differences before multiple comparisons correction. (B) The difference between coefficients found for changes to connections within the seizure onset zone versus outside it.

ence of an IED and the spread of an IED tend to be significant and positive but weaker (Fig. $4 \mathbf{A}$; Spearman's correlation, Bonferroni-corrected $p$-values $(n=16)$ mean $r_{A E C}=0.45+/-0.073$, mean $r_{i C o h}=0.42+/-0.070$, mean $r_{i P L V}=0.47+/-0.13, r_{x c o r r}=0.48, r_{A R}=0.44$, all $p<8.47 \times 10^{-3}$ ), and the correlation between the number of IEDs and the spread of IEDs tends to be nonsignificant and much weaker (Fig. $4 \mathbf{A}$; Spearman's correlation, Bonferroni-corrected $p$-values $(n=16)$ mean $r_{A E C}=0.048+/-0.083$, mean $r_{i C o h}=0.086+/-0.12$, mean $r_{i P L V}=0.058+/-0.16, r_{x c o r r}=0.31, r_{A R}=0.12$; only broadband cross-correlation and theta imaginary phase-locking value had signififcant $p$-values after multiple comparisons correction). Due to the lack of consistent changes in functional connectivity resulting from the number of IEDs and the predictor's high similarity with the presence of an IED, we excluded this predictor from further analysis.

Next, we sought to characterize similarities between each band-measure combination. Here we test three hypotheses. First, we hypothesize that coefficients will be similar across measures within a given frequency band. 
Our second hypothesis is that coefficients will be similar within a measure, across frequency bands. Our third hypothesis is that there will be a patterned similarity across bands. Specifically, the low frequencies (theta, alpha, and beta) will be similar to each other, but dissimilar to high frequencies (gamma and high gamma) and vice versa. High and low frequencies are often described in the literature as showing opposing patterns, and are theoretically proposed to have opposite roles [18. To test these three hypotheses, we calculate similarity matrices for each predictor. Each element of the similarity matrix is the Spearman's correlation between the distribution of functional connectivity changes across subjects for two band-measure combinations. We show these matrices sorted by frequency band and by measure (Fig. 4 $\mathbf{B}$ ).

To test our first hypothesis, we correlate the upper triangles of these pairs of similarity matrices with masks that operationalize each hypothesis. For example, in the band similarity hypothesis, the mask has ones for entries from the same band and zeros for entries from different bands. To test the significance of our findings, we compare the correlations calculated from empirical data to correlations calculated from 100 similarity matrices generated from data where band and measure labels were permuted within subjects. We find similar correlations to the frequency band mask for both the spread and presence of IEDs (Fig. 4 C ; Pearson's correlation $(n=3)$ $\left.r_{\text {presence }}(112)=0.35, r_{\text {spread }}(112)=0.30\right)$, although the correlation is slightly stronger for the presence of IEDs. Both correlations were stronger than all 100 correlations obtained from permuted null models (Fig. S8). To test our second hypothesis, we repeated the same analysis, but with a measure mask. Here, we find that correlations are strongest for the spread-of-IEDs predictor (Fig. 4C; Pearson's correlation $r_{\text {presence }}(112)=0.26$, $\left.r_{\text {spread }}(112)=0.38\right)$. Here, the correlation for both predictors was larger than all 100 null models (Fig S7). To test our third hypothesis, we repeated the same analysis with a grouped frequency (high or low) mask. Here, we find much larger correlations in the presence of IEDs (Fig. 4C; Pearson's correlation $r_{\text {presence }}(112)=0.59$, $\left.r_{\text {spread }}(112)=0.37\right)$. Additionally, this mask explained much more variance than each band individually for the presence, but not spread, of IEDs. Here, the correlation for both predictors was larger than 100 null models (Fig S7). Ultimately, we find that both the bands and measures tend to have similar effects. Additionally, the presence of IEDs seems to show more similar changes to functional connectivity within bands than within measures, although those effects differ between high and low frequencies.

\section{Explanatory Sources of Individual Variability}

We next sought to understand sources of individual variability in changes to connectivity. However, partic- ipants in this study have coverage over a unique and heterogeneous set of regions, making it difficult to determine whether differences across individuals are due to differences in coverage or in characteristics of the participants. To test whether changes across subjects may be due to differences in electrode placement, we wished to quantify whether connectivity changes were more similar within individuals or within regions. To quantify similarity, we calculated the Spearman's correlation between the vector of connectivity changes across all bands, measures, and predictors between each individual contact (Fig. $\mathbf{5} \mathbf{A B})$. We then calculated the correlation between these similarity matrices and masks selecting for entries from either the same individual or the same region. Here, regions are clinician-provided labels for each contact. We find a much larger Pearson's $r$ value for the subject mask, compared to the regions mask (Pearson's correlation $r_{s u b j}(2,401,318)=0.13$, $\left.r_{\text {region }}(2,401,318)=2.21 \times 10^{-3}\right)$. This observation motivated us to continue investigating individual, rather than regional, differences in changes to connectivity resulting from IEDs.

We next tested whether differences in electrode type mediated these individual differences. Some individuals in the cohort were implanted with only flat grid or strip electrodes on the surface of the brain, while others were implanted with only penetrating or depth electrodes that reached deeper structures. These two types of electrodes sample the brain differently, and can record from different neural structures. We hypothesized that these differences in sampling might lead to different magnitudes of changes to functional connectivity. However, we found that no band-measure combination showed a statistically significant main effect for electrode type after multiple comparisons correction (Fig. S9; permutation-based linear model).

We next tested which participant-specific features would best predict changes to connectivity for each band and measure using group LASSO regression. Group LASSO applies a penalty to groups of coefficients in a regression equation, allowing coefficients that do not greatly increase the explanatory power of the model to be regularized to zero. A parameter $\lambda$ scales the regularization, such that a larger $\lambda$ will result in a stricter penalty. Here, we chose the value of $\lambda$ that minimized mean squared error across leave-one-out cross validation.

We hypothesized that behavioral, clinical, contact, and demographic factors might impact the magnitude of IED effects on functional connectivity. We include one behavioral variable, which is the average task performance across a battery of 5 possible free recall and spatial navigation tasks completed by each participant. We include 3 clinical variables: (1) presence or absence of lesion, (2) age at seizure onset, and (3) the underlying etiology of epilepsy. We include 5 demographic variables: (1) age, (2) race, (3) sex, (4) handedness, and (5) institution. Lastly, we include 3 contact variables: (1) the cognitive system [47] that contains the contact with the 
A

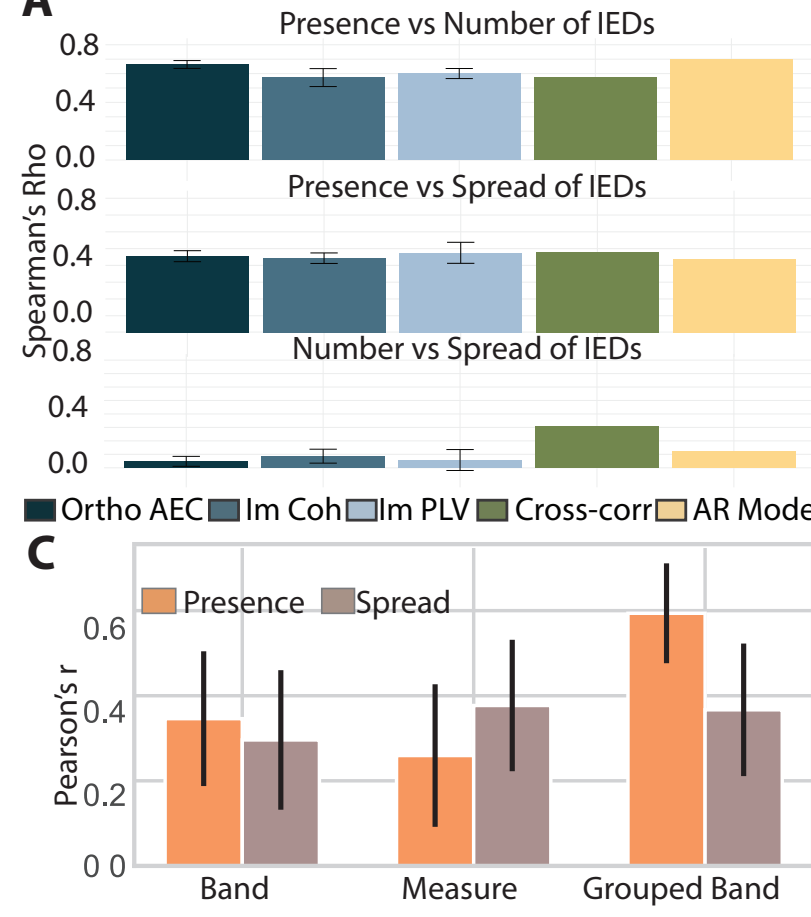

B
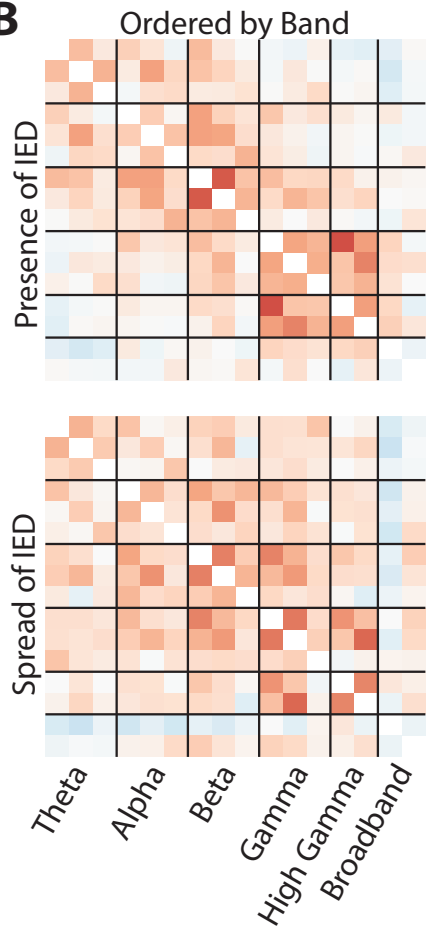
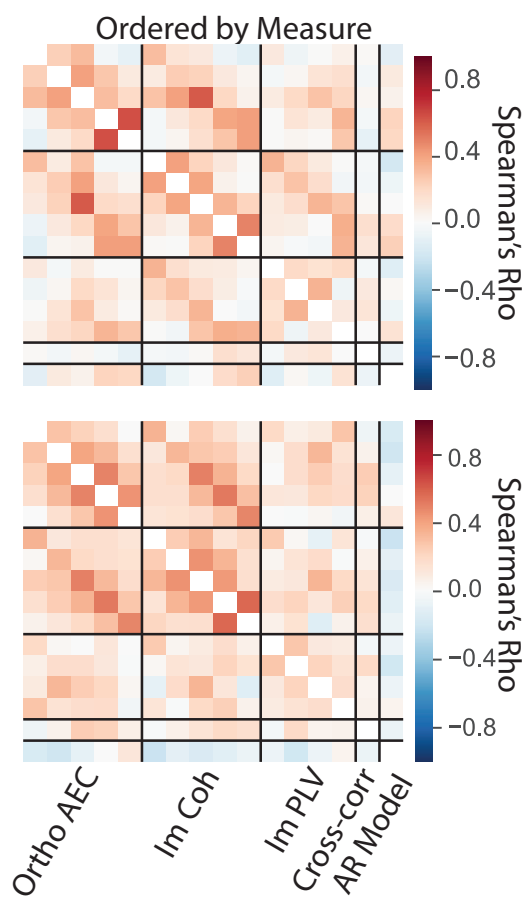

FIG. 4. Similarity across predictors, measures, and bands. (A) Spearman's correlations between predictors. A bar plot showing the average correlation and standard error for each measure across bands. Error bars are the standard error across bands, when there are multiple bands to test. Colors indicate different measures. (B) Similarity matrices between all band-measure combinations. (Left) Matrices ordered by band. (Right) Matrices ordered by measure. (C) Correlations with a frequency band mask (left), measure mask (middle), and frequency band group, i.e. high or low (right) for each predictor. Error bars show the $95 \%$ confidence interval for each correlation.

most IEDs, (2) the hemisphere that contains the contact with the most IEDs, and (3) whether the participant had grid contacts, depth contacts, or both. Fifty-eight participants had all these fields of information.

We restrict our analyses to only measures that had changes in connectivity significantly different from 0 in both IED detectors: the orthogonal amplitude envelope correlation, and imaginary coherence. The variables and corresponding regularized coefficients included in each grouped LASSO regression are shown in Fig. $\mathbf{5}$ C. For categorical variables, the largest beta value assigned to a contrast at a given level was used. For both the presence and spread of IEDs, we found that some bands and measures were not well explained by any of the included variables. However, all variables except the average task performance and presence of lesion were included at least once. The most commonly included variable across both predictors was the cognitive system containing most IEDs (i.e., the IED locus). This variable was included in 5 out of 7 models that retained variables. Additionally, we found that the hemisphere that contains most IEDs, the underlying etiology, as well as the age, race and institution are common across both IED features. Overall, the models for the presence of IEDs spanned a wider range of bands, and included a larger number of variables than those for the spread. We also noted some differences between models explaining changes in the presence versus spread of IEDs. The type of contact used (grid, depth, or mixed) and the age of seizure onset were commonly included variables for the presence, but not spread of IEDs.

\section{DISCUSSION}

Here, we complete a thorough quantification of the changes in functional connectivity associated with IEDs in a large sample of 145 individuals. We test 5 different frequency bands and the broadband signal for changes associated with 5 different measures of functional connectivity and with 3 properties of IEDs. One key insight from our study is that, despite the tremendous heterogeneity in IED properties both within and between individuals [33, 51, we observe consistent increases in functional connectivity associated with IEDs. Our study allows us to determine which properties of IEDs are responsible for the observed changes. We find that, once a single IED has occurred, more IEDs within a time 
A

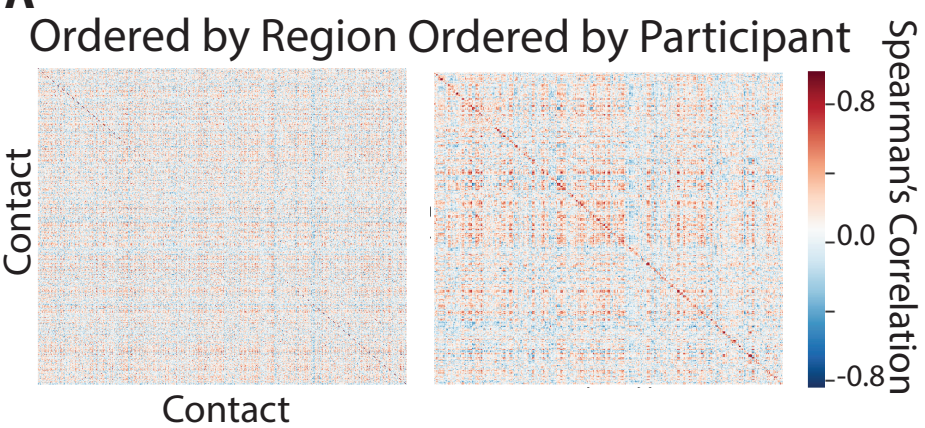

C

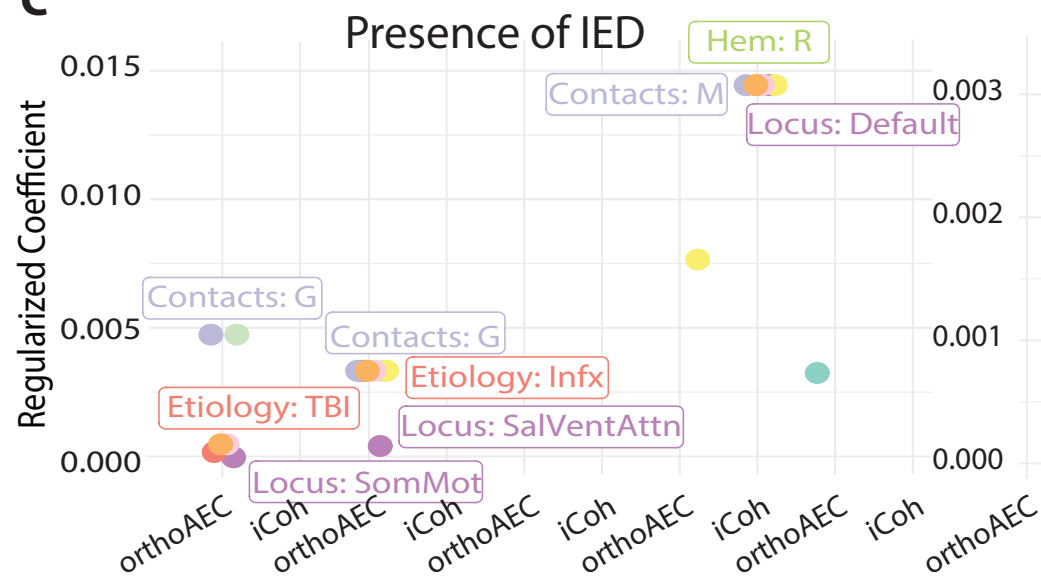

B

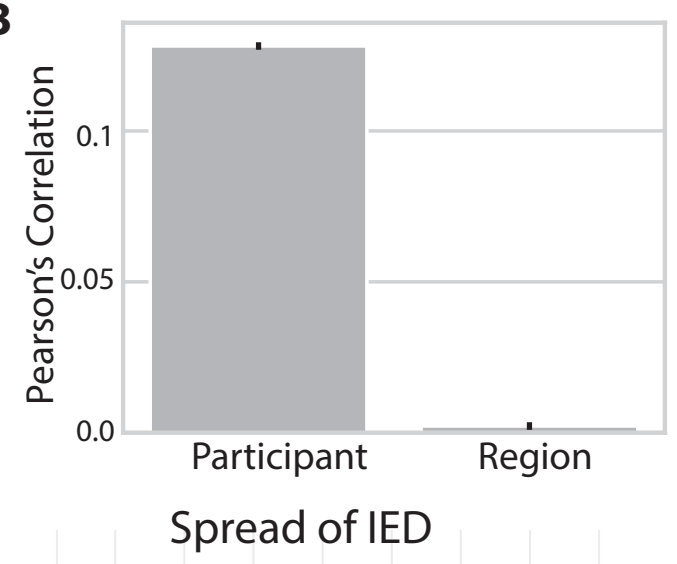

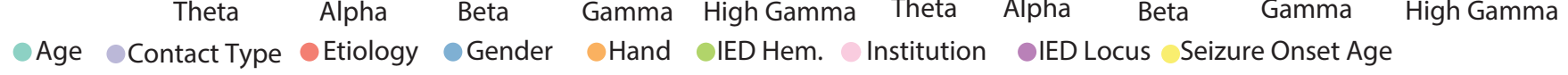

FIG. 5. Individual differences. (A) Similarity matrices for coefficient distributions across all predictors, bands, and measures, but between contacts. (Left) Matrices ordered by region. (Right) Matrices ordered by participant. (B) Correlations for masks selecting for participants, or regions. Error bars are the $95 \%$ confidence interval for each correlation. (C) Predictors included for each model, and their corresponding regularized coefficient. For categorical variables representing clinical, or contact level variables, text is displayed showing the level of the categorical variable that had the largest coefficient. Different variables are shown in different colors, with a legend matching colors to variables at the bottom of the plot. Only included variables are shown in the legend; therefore, no color is assigned to the average task performance, or an indicator of whether of not each participant was lesional. Legend and text abbreviations are as follows: Hem. is an abbreviation for hemisphere, and $R$ indicates right; Contacts $G$ indicates grid coverage, and $M$ indicates mixed coverage; Etiology TBI indicates traumatic brain injury, and Infx indicates infection; Locus SalVentAttn indicates the salience and ventral attention network, SomMot indicates the somatomotor network, and DorsAttn indicates the dorsal attention network.

window do not further disrupt ongoing global functional connectivity, whereas IEDs that spread to more contacts further increase connectivity. Additionally, individuals whose functional connectivity changed significantly in response to the presence of an IED tended to show large changes associated with the spread of IEDs. Our work reveals that only amplitude-influenced measures (i.e. orthogonal amplitude envelope correlations and imaginary coherence) show changes, whereas phase-based and broadband measures tend to remain more stable. This result is consistent with work showing that these two dimensions of statistical association between timeseries are not biologically redundant [52. Across bands, we see that effects tend to be similarly sized within low and high frequency groups individually, but not across them. We also observe a high degree of individual variability in changes to functional connectivity associated with IEDs. No single feature robustly explained the variation observed across all measures and bands, but the cognitive system that IEDs originate from explains the most variance. The changes reported here contribute substantial evidence towards an important unanswered question in epilepsy research regarding the impact of IEDs on functional connectivity. Therefore, these results have important implications for preprocessing, analyses, and interpretation of research conducted with human 
iEEG data.

\section{IEDs disrupt multiple physiological processes}

Our results show that responses to the presence of IEDs are more similar within groups of high and low frequencies than with each band individually. While we cannot infer the mechanisms that generate oscillations from their frequency bands alone, previous research has reported differing roles for oscillations in low- versus highfrequency bands. For example, early intracranial recordings show that lower frequency oscillations $(<30 \mathrm{~Hz})$ tend to be much more spatially distributed than higher frequency oscillations [21, 53. Further work has shown that power in the highest frequency band investigated here, high gamma, can be correlated with localized spiking activity [20, 22]. Additionally, while low frequency signals are thought to influence top-down cognitive processes, high frequency signals are thought to reflect bottom-up sensory driven processing [54, [55]. Here, we provide evidence that each of these processes is disrupted during IEDs, but might be impacted differently.

Our results point to two important future directions. First, it would be of interest to investigate changes in cross-frequency interactions and the slope of the power spectral density associated with IEDs. High and low frequencies have also been shown to have a preferential interaction direction, where the amplitude of higher frequencies is modulated by the phase of lower frequencies [18. While these interactions can be spurious and require strict testing for the presence of oscillations above the $1 / \mathrm{f}$ background and against null models that preserve autocorrelation 556, 57, a detailed investigation of which of these interactions are changed during IEDs would help further elucidate the neurophysiological processes that they disrupt. Second, opposing changes in high- and low-frequency bands can sometimes be explained by changes in the slope of the $1 / \mathrm{f}$ background, rather than changes in each band individually [58. In studies of neural activation, the slope and power in specific bands are directly and intuitively related, however it is unclear if such a relationship exists for connectivity. Additionally, here we do not see strongly anti-correlated patterns between low and high frequencies, and therefore we do not think this effect is driving the observed patterns. However, investigating changes in the slope of the 1 /f background, as well as other non-sinusoidal features of the signal, would also add significantly to our understanding of how IEDs impact ongoing physiology.

\section{Implications for iEEG researchers}

Our work has important implications for researchers who use iEEG data to understand basic features of the human brain that are independent of epilepsy and cannot be answered with other methods 59. There exist multiple methods for discounting the impact of IEDs on this type of research, including removing time points with IEDs, removing channels with IEDS, or including IED data. Here, we observe statistically significant increases in functional connectivity in a sample of individuals an order of magnitude larger than a typical iEEG study. The large-group effects extended beyond the contacts that showed IEDs. Additionally, some individuals showed large changes in functional connectivity associated with IEDs. Given that iEEG research is well-suited to within-individual experimental designs, we recommend, based on our results, that researchers remove entire time points that contain IEDs, rather than simply the channels that contain frequent IEDs. This approach will likely make results obtained from this population more consistent with the noninvasive EEG and fMRI literature in non-epileptic populations, and will likely lead to more robust inferences about generalized processes not linked to epilepsy.

\section{Unexplained variance in connections and individuals}

Here, we test a few simple hypotheses about which connections might be contributing to increases in functional connectivity. Specifically, we test if increases in functional connectivity are preferentially explained by (1) the weakest edges in the distribution, (2) connections in the seizure onset zone, (3) between regions in the IZ, (4) between grid and depth contacts, and (5) between grey and white matter contacts on depth electrodes.

The most consistent feature identified was that functional connectivity within pathological tissue, either the $\mathrm{SOZ}$ or IZ is increased more when IEDs spread to more contacts. The largest effects are seen in higher frequency bands, which are thought to reflect more local activity. This pattern of findings is intuitive, given that the IEDs likely spread to regions within that pathological tissue. While edges outside these pathological regions still increase with broader spread of IEDs, this evidence suggests that the spread might be impacting more spatially local networks. However, none of the edge subsets we explored showed larger connectivity increases for the presence of IEDs. This observation is particularly noteworthy for grey and white matter contacts within depth electrodes. White matter has sometimes been considered to not have much meaningful signal, and be more appropriately used as a reference for recordings from grey matter 60]. However, here we see similar changes to functional connectivity in metrics that attempt to control for volume conducted signal, consistent with work suggest- 
ing that these contacts might be recording some signals from distant grey matter not recorded elsewhere 61 .

Interictal connectivity changes from healthy controls show complex patterns of increases and decreases across systems 62, 63. Additionally, these changes are temporally dynamic, but culminate in broad increases in connectivity during seizure initiation 24. Given that IEDs are often considered to be a simple instantiation of epileptic activity that leads to seizures 4 , the specific connections contributing to increases likely vary depending on the specific seizure semiology features of the individual.

Given the large variability in changes in functional connectivity across individuals, we also sought to characterize which features of individual participants explained some of the observed variance. The most consistently included variable was the cognitive system that most IEDs originated from. The system from which IEDs originate might be capturing information regarding the specific location of the seizure onset zone of a given individual and the associated epilepsy syndrome, i.e. frontal or temporal lobe epilepsy. Disentangling the contributions of these two features presents an exciting direction for future work. While we did not have access to each participants epilepsy syndrome directly, we expect these different phenotypes to show different patterns, due to the diverse spatial distribution of pathology and connectivity changes from controls 62 64. Additionally, theoretical work on the spread of perturbations in brain networks suggests different directions of travel and patterns for perturbations originating in different regions 65, 66. It is thus likely that both clinical and generalizable neural features underlie the explanatory power of the cognitive system where the IED originated.

While it is unsurprising that no single variable captured individual variability well, it is worth considering that some band-measure combinations were not wellexplained by any combination of variables. However, the cohort used in this study was not homogeneous, and contains several additional sources of interindividual differences that are not captured by our variables. For example, we included no information about the shape or frequency of IEDs, treatment plans while in the epilepsy monitoring unit, or the type of epilepsy. A meta-analysis of smaller studies providing this information might better be able to address individual variability in observed effects.

\section{Limitations}

This study presented a thorough characterization of how global functional connectivity changes during IEDs, and simplified the space of possible biophysical interactions that are affected during an IED. However, the findings from this study should be interpreted in light of limitations in our approach and methods. First, we sought to characterize changes to the macroscale behav- ior of the system, quantified as global connectivity. However, this approach prevents us from identifying even robust local changes in connections that counteract the shift in mean connection weight. Second, we chose to investigate a window size of 1 second, but it is possible that other time windows could capture complementary changes across different frequency bands. Third, we used a permutation-based linear model to estimate effect sizes, which precludes an identification of nonlinear effects associated with any of our predictors. Fourth, we tested for changes in the magnitude of functional connectivity metrics, but this approach creates inconsistency for metrics that use negative values (AR model, amplitude envelope correlation) by preventing the identification of changes that shift from large positive to large negative values.

One important final consideration is the potential for filtering artifacts to influence our results[28]. Filters, like those used when bandpass filtering data, can induce spurious oscillations and estimates of connectivity when applied to sharp transient activity or steps. For transparency, we show the results of applying the filters used in our preprocessing steps in Fig. S2. We are confident that our results are not strongly influenced by this type of artifact for the following reasons: (1) the lack of strong changes in phase-locking value associated with IEDs, (2) the significant changes to connectivity in contacts outside the IZ, and (3) the significant differences seen in functional connectivity only from contacts with no IEDs. However, it is still possible that these artifacts could impact our findings in small ways.

\section{Conclusions and Future Directions}

In addition to future studies investigating other aspects of neural activity, this exploratory work facilitates several avenues of hypothesis-driven research. While we were explicitly looking for large effects that could be visible after stringent multiple comparisons correction, specific hypotheses about more homogeneous groups that are subsets of this population could elucidate more subtle changes. For example, frontal versus temporal lobe epilepsy cases have been show to have changes to functional connectivity in different systems at rest 62, 63. Future work could test whether the presence or spread of IEDs impacts interactions between those systems selectively, or whether changes are only seen in resting-state activity. Additionally, while work has already shown a relationship between the presence of IEDs and task performance, an extension of this work to task data could assess whether that relationship extends to other features of IEDs such as their number and spread. Lastly, specific etiologies can have more homogeneous neural abnormalities. It would be interesting to investigate whether deviations from the average profile presented here are localized to regions of pathology.

In this work, we demonstrate consistent increases in 
amplitude-influenced functional connectivity associated with IEDs. We present evidence that IEDs influence multiple features of neurophysiology and that the observed changes in functional connectivity are not limited to pathological tissue. Based on these observations, we recommend that basic scientists working with iEEG data who wish to make claims about the general, nonepileptic population using functional connectivity remove time points including IEDs from their analysis. Additionally, these observations demonstrate that the epileptic brain displays a tendency towards synchrony in the context of small perturbations, even when that synchrony does not amount to a seizure. Future work investigating and modeling the dynamics of seizures can now use these observations to better understand the principles underlying seizure generation.

\section{CITATION DIVERSITY STATEMENT}

Recent work in several fields of science has identified a bias in citation practices such that papers from women and other minority scholars are under-cited relative to the number of such papers in the field 67-71]. Here we sought to proactively consider choosing references that reflect the diversity of the field in thought, form of contribution, gender, race, ethnicity, and other factors. First, we obtained the predicted gender of the first and last author of each reference by using databases that store the probability of a first name being carried by a woman [71, 72]. By this measure (and excluding self-citations to the first and last authors of our current paper), our references contain 8.6\% woman(first)/woman(last), $15.7 \% \mathrm{man} /$ woman, $15.7 \%$ woman $/ \mathrm{man}$, and $58.6 \% \mathrm{man} / \mathrm{man}$. This method is limited in that a) names, pronouns, and social media profiles used to construct the databases may not, in every case, be indicative of gender identity and b) it cannot account for intersex, non-binary, or transgender people. Second, we obtained predicted racial/ethnic category of the first and last author of each reference by databases that store the probability of a first and last name being carried by an author of color [? ]. By this measure (and excluding self-citations), our references contain $9.42 \%$ author of color (first)/author of color(last), $12.78 \%$ white author/author of color, $24.31 \%$ author of color/white author, and $53.49 \%$ white author/white author. This method is limited in that a) names and Florida Voter Data to make the predictions may not be indicative of racial/ethnic identity, and b) it cannot account for Indigenous and mixed-race authors, or those who may face differential biases due to the ambiguous racialization or ethnicization of their names. We look forward to future work that could help us to better understand how to support equitable practices in science.

\section{ACKNOWLEDGEMENTS}

D.S.B. and J.S. acknowledge support from the John D. and Catherine T. MacArthur Foundation, the Alfred P. Sloan Foundation, the ISI Foundation, the Paul Allen Foundation, the Army Research Laboratory (W911NF-10-2-0022), the Army Research Office (Bassett-W911NF-14-1-0679, Grafton-W911NF-161-0474, DCIST- W911NF-17-2-0181), the Office of Naval Research, the National Institute of Mental Health (2R01-DC-009209-11, R01 - MH112847, R01-MH107235, R21-M MH-106799), the National Institute of Child Health and Human Development (1R01HD086888-01), National Institute of Neurological Disorders and Stroke (R01 NS099348), and the National Science Foundation (BCS-1631550, IIS-1926757). The content is solely the responsibility of the authors and does not necessarily represent the official views of any of the funding agencies.
[1] J. J. Falco-Walter, I. E. Scheffer, and R. S. Fisher, The new definition and classification of seizures and epilepsy, Epilepsy Research 139, 73 (2018).

[2] R. S. Fisher, C. Acevedo, A. Arzimanoglou, A. Bogacz, J. H. Cross, C. E. Elger, J. Engel, L. Forsgren, J. A. French, M. Glynn, D. C. Hesdorffer, B. I. Lee, G. W. Mathern, S. L. Moshé, E. Perucca, I. E. Scheffer, T. Tomson, M. Watanabe, and S. Wiebe, ILAE Official Report: A practical clinical definition of epilepsy, Epilepsia 55, 475 (2014)

[3] M. Avoli, Do interictal discharges promote or control seizures? Experimental evidence from an in vitro model of epileptiform discharge, Epilepsia 42, 2 (2001)

[4] D. Prince and B. Connors, Mechanisms of interictal epileptogenesis., Advances in neurology 44, 275 (1986).

[5] Ung Haomeng, Cazares C, Nanivadekar A, Kini L, Wagenaar J, Becker D, Krieger A, L. T. Litt, and Davis Kathryn, Interictal Epileptiform Activity Outside the Seizure Onset Zone Impacts Cognition, Brain 18, 2157
(2017)

[6] A. Aldenkamp and J. Arends, The Relative Influence of Epileptic EEG Discharges, Short Nonconvulsive Seizures, and Type of Epilepsy on Cognitive Function, Epilepsia 45, 54 (2004).

[7] J. Vannest, J. R. Tenney, R. Gelineau-Morel, T. Maloney, and T. A. Glauser, Cognitive and behavioral outcomes in benign childhood epilepsy with centrotemporal spikes, Epilepsy and Behavior 45, 85 (2015).

[8] P. Dahal, N. Ghani, A. Flinker, P. Dugan, D. Friedman, W. Doyle, O. Devinsky, D. Khodagholy, and J. N. Gelinas, Interictal epileptiform discharges shape large-scale intercortical communication, Brain 10.1093/brain/awz269 (2019).

[9] C. J. Keller, W. Truccolo, J. T. Gale, E. Eskandar, T. Thesen, C. Carlson, O. Devinsky, R. Kuzniecky, W. K. Doyle, J. R. Madsen, D. L. Schomer, A. D. Mehta, E. Halgren, and S. S. Cash, Heterogeneous neuronal firing patterns during interictal epileptiform discharges in 
the human cortex, A JOURNAL OF NEUROLOGY 10, $19(2020)$

[10] E. A. Shamshiri, T. M. Tierney, M. Centeno, K. St Pier, R. M. Pressler, D. J. Sharp, S. Perani, J. H. Cross, and D. W. Carmichael, Interictal activity is an important contributor to abnormal intrinsic network connectivity in paediatric focal epilepsy, Human Brain Mapping 38, 221 (2017)

[11] P. J. van Houdt, P. P. Ossenblok, A. J. Colon, K. H. Hermans, R. M. Verdaasdonk, P. Boon, and J. C. de Munck, engAre epilepsy-relalated fmri components dependent on the presence of interictal epileptic discharges in scalp eeg?, BRAIN TOPOGRAPHY 28, 606 (2015).

[12] H. Laufs, K. Hamandi, A. Salek-Haddadi, A. K. Kleinschmidt, J. S. Duncan, and L. Lemieux, Temporal lobe interictal epileptic discharges affect cerebral activity in "default mode" brain regions, Human Brain Mapping 28, $1023(2007)$

[13] X. Tong, D. An, F. Xiao, D. Lei, R. Niu, W. Li, J. Ren, W. Liu, Y. Tang, L. Zhang, B. Zhou, Q. Gong, and D. Zhou, Real-time effects of interictal spikes on hippocampus and amygdala functional connectivity in unilateral temporal lobe epilepsy: An EEG-fMRI study, Epilepsia 60, 246 (2019).

[14] R. Lopes, F. Moeller, P. Besson, F. Ogez, W. Szurhaj, X. Leclerc, M. Siniatchkin, M. Chipaux, P. Derambure, and L. Tyvaert, Study on the relationships between intrinsic functional connectivity of the default mode network and transient epileptic activity, Frontiers in Neurology 5, 1 (2014)

[15] J. Courtiol, M. Guye, F. Bartolomei, S. Petkoski, and V. K. Jirsa, Dynamical mechanisms of interictal restingstate functional connectivity in epilepsy, The Journal of Neuroscience, JN (2020)

[16] K. MacLeod, A. Backer, and G. Laurent, Who read temporal information contained across synchronized and oscillatory spike trains?, Nature 255, 243 (1998).

[17] M. Schneider, B. Dann, S. Sheshadri, H. G. Scherberger, and M. Vinck, A general theory of coherence between brain areas, bioRxiv , 2020.06.17.156190 (2020).

[18] O. Jensen and L. L. Colgin, Cross-frequency coupling between neuronal oscillations, Trends in Cognitive Sciences 11, 267 (2007)

[19] J. Riddle, K. Hwang, D. Cellier, S. Dhanani, and M. D'esposito, Causal evidence for the role of neuronal oscillations in top-down and bottom-up attention, Journal of Cognitive Neuroscience 31, 768 (2019).

[20] M. Leszczyński, A. Barczak, Y. Kajikawa, I. Ulbert, A. Y. Falchier, I. Tal, S. Haegens, L. Melloni, R. T. Knight, and C. E. Schroeder, Dissociation of broadband high-frequency activity and neuronal firing in the neocortex, Science Advances 6, 1 (2020).

[21] N. Crone, D. L. Miglioretti, B. Gordon, and R. P. Lesser, Functional mapping of human sensorimotor cortex with electrocorticographic spectral analysis, Brain 121, 2301 (1998)

[22] J. Manning, J. Jacobs, I. Fried, and M. Kahana, Broadband Shifts in Local Field Potential Power Spectra Are Correlated with Single-Neuron Spiking in Humans, Journal of Neuroscience 29, 13613 (2009).

[23] Y. Ezzyat, J. E. Kragel, J. F. Burke, D. F. Levy, A. Lyalenko, P. Wanda, L. O'Sullivan, K. B. Hurley, S. Busygin, I. Pedisich, M. R. Sperling, G. A. Worrell, M. T. Kucewicz, K. A. Davis, T. H. Lucas, C. S. Inman,
B. C. Lega, B. C. Jobst, S. A. Sheth, K. Zaghloul, M. J. Jutras, J. M. Stein, S. R. Das, R. Gorniak, D. S. Rizzuto, and M. J. Kahana, Direct Brain Stimulation Modulates Encoding States and Memory Performance in Humans, Current Biology 27, 1251 (2017).

[24] S. P. Burns, S. Santaniello, R. B. Yaffe, C. C. Jouny, N. E. Crone, G. K. Bergey, W. S. Anderson, and S. V. Sarma, Network dynamics of the brain and influence of the epileptic seizure onset zone, Proceedings of the National Academy of Sciences of the United States of America 111, E5321 (2014).

[25] E. L. Juárez-Martinez, I. A. Nissen, S. Idema, D. N. Velis, A. Hillebrand, C. J. Stam, and E. C. van Straaten, Virtual localization of the seizure onset zone: Using noninvasive MEG virtual electrodes at stereo-EEG electrode locations in refractory epilepsy patients, NeuroImage: Clinical 19, 758 (2018)

[26] L. L. W. Owen, A. C. Heusser, and J. R. Manning, A Gaussian process model of human electrocorticographic 10.1101/121020.

[27] R. F. Betzel, J. D. Medaglia, A. E. Kahn, J. Soffer, D. R. Schonhaut, and D. S. Bassett, Inter-regional ECoG correlations predicted by communication dynamics, geometry, and correlated gene expression, , 1 (2017), arXiv: 1706.06088

[28] A. de Cheveigné and I. Nelken, Filters: When, Why, and How (Not) to Use Them, Neuron 102, 280 (2019)

[29] M. W. Brown Iii, B. E. Porter, D. J. Dlugos, J. Keating, A. B. Gardner, P. B. B. Storm, and E. D. Marsh, Comparison of novel computer detectors and human performance for spike detection in intracranial EEG 10.1016/j.clinph.2007.04.017 (2007).

[30] N. Gaspard, L. J. Hirsch, S. M. LaRoche, C. D. Hahn, and B. Westover, Interrater agreement for Critical Care EEG Terminology, Epilepsia 55, 1366 (2014).

[31] R. Janca, P. Jezdik, R. Cmejla, M. Tomasek, G. A. Worrell, M. Stead, J. Wagenaar, J. G. Jefferys, P. Krsek, V. Komarek, P. Jiruska, and P. Marusic, Detection of Interictal Epileptiform Discharges Using Signal Envelope Distribution Modelling: Application to Epileptic and Non-Epileptic Intracranial Recordings, Brain Topography 28, 172 (2014)

[32] Y. Lai, W. Van Drongelen, K. Hecox, D. Frim, M. Kohrman, and B. He, Cortical activation mapping of epileptiform activity derived from interictal ECoG spikes, Epilepsia 48, 305 (2007).

[33] E. C. Conrad, S. B. Tomlinson, J. N. Wong, K. F. Oechsel, R. T. Shinohara, B. Litt, K. A. Davis, and E. D. Marsh, Spatial distribution of interictal spikes fluctuates over time and localizes seizure onset, Brain 143, 554 (2020)

[34] S. B. Tomlinson, C. Bermudez, C. Conley, M. W. Brown, B. E. Porter, and E. D. Marsh, Spatiotemporal mapping of interictal spike propagation: A novel methodology applied to pediatric intracranial eeg recordings, Frontiers in Neurology 7, 1 (2016).

[35] N. Roehri, F. Pizzo, F. Bartolomei, F. Wendling, and C.G. Bé Nar, What are the assets and weaknesses of HFO detectors? A benchmark framework based on realistic simulations 10.1371/journal.pone.0174702 (2017).

[36] R. Oostenveld, P. Fries, E. Maris, and J. M. Schoffelen, FieldTrip: Open source software for advanced analysis of MEG, EEG, and invasive electrophysiological data, Computational Intelligence and Neuroscience 
2011, 10.1155/2011/156869 (2011).

[37] W. Singer, Cortical dynamics revisited (2013).

[38] I. Toni, Interactions between posterior gamma and frontal alpha/beta oscillations during imagined actions, Frontiers in Human Neuroscience 2, 1 (2008).

[39] J. F. Hipp, D. J. Hawellek, M. Corbetta, M. Siegel, and A. K. Engel, Large-scale cortical correlation structure of spontaneous oscillatory activity, Nature Neuroscience 15, $884(2012)$

[40] G. Nolte, E. Galindo-Leon, Z. LI, X. Liu, and A. K. Engel, Mathematical relations between measures of brain connectivity estimated from electrophysiological recordings for Gaussian distributed data, bioRxiv , 680678 (2019)

[41] R. Bruña, F. Maestú, and E. Pereda, Phase locking value revisited: Teaching new tricks to an old dog, Journal of Neural Engineering 15, 10.1088/1741-2552/aacfe4 (2018), arXiv:1710.08037

[42] A. M. Bastos and J. M. Schoffelen, A tutorial review of functional connectivity analysis methods and their interpretational pitfalls, Frontiers in Systems Neuroscience 9, 175 (2016)

[43] K. Subhash Chandran, A. Mishra, V. Shirhatti, and S. Ray, Comparison of matching pursuit algorithm with other signal processing techniques for computation of the time-frequency power spectrum of brain signals, Journal of Neuroscience 36, 3399 (2016).

[44] T. Schneider and A. Neumaier, Algorithm 808: ARfita matlab package for the estimation of parameters and eigenmodes of multivariate autoregressive models, ACM Transactions on Mathematical Software 27, 58 (2001).

[45] G. Solovey, L. M. Alonso, T. Yanagawa, N. Fujii, M. O. Magnasco, G. A. Cecchi, and A. Proekt, Loss of Consciousness Is Associated with Stabilization of Cortical Activity, Journal of Neuroscience 35, 10866 (2015).

[46] A. Schaefer, R. Kong, E. M. Gordon, T. O. Laumann, X.N. Zuo, A. J. Holmes, S. B. Eickhoff, and B. T. T. Yeo, Local-Global Parcellation of the Human Cerebral Cortex from Intrinsic Functional Connectivity MRI, Cerebral Cortex 28, 3095 (2018)

[47] T. Yeo, F. M. Krienen, J. Sepulcre, M. R. Sabuncu, D. Lashkari, M. Hollinshead, J. L. Roffman, J. W. Smoller, L. Zöllei, J. R. Polimeni, B. Fischl, H. Liu, and R. Buckner, The organization of the human cerebral cortex estimated by intrinsic functional connectivity, Journal of neurophysiology 106, 1125-1165 (2011)

[48] A. Klein, T. D. Canton, S. S. Ghosh, B. Landman, J. Lee, A. Worth, and T. Dal Canton, Open labels: online feedback for a public resource of manually labeled brain images, 16th Annual Meeting for the Organization of $\mathrm{Hu}-$ man Brain Mapping. , 84358 (2010).

[49] J. Jacobs, J. Miller, S. A. Lee, T. Coffey, A. J. Watrous, M. R. Sperling, A. Sharan, G. Worrell, B. Berry, B. Lega, B. C. Jobst, K. Davis, R. E. Gross, S. A. Sheth, Y. Ezzyat, S. R. Das, J. Stein, R. Gorniak, M. J. Kahana, and D. S. Rizzuto, Direct Electrical Stimulation of the Human Entorhinal Region and Hippocampus Impairs Memory, Neuron 92, 983 (2016).

[50] K. Sainani, The Importance of Accounting for Correlated Observations, PM and R 2, 858 (2010).

[51] R. J. Quon, S. Meisenhelter, R. H. Adamovich-Zeitlin, Y. Song, S. A. Steimel, E. J. Camp, M. E. Testorf, T. A. MacKenzie, R. E. Gross, B. C. Lega, M. R. Sperling, M. J. Kahana, and B. C. Jobst, Factors correlated with intracranial interictal epileptiform discharges in refractory epilepsy, Epilepsia , 1 (2020)

[52] M. Siems and M. Siegel, Dissociated cortical phase- and amplitude-coupling patterns in the human brain, bioRxiv $10.1101 / 485599$ (2018).

[53] N. E. Crone, D. L. Miglioretti, B. Gordon, J. M. Sieracki, M. T. Wilson, S. Uematsu, and R. P. Lesser, Functional mapping of human sensorimotor cortex with electrocorticographic spectral analysis: I. Alpha and beta eventrelated desynchronization, Brain 121, 2271 (1998)

[54] W. Singer, A. K. Engel, and P. Fries, Nature Reviews. Neuroscience, Tech. Rep. 10 (2001).

[55] C. G. Richter, W. H. Thompson, C. A. Bosman, and P. Fries, Top-down beta enhances bottom-up gamma, Journal of Neuroscience 37, 6698 (2017)

[56] E. M. Gerber, B. Sadeh, A. Ward, R. T. Knight, and L. Y. Deouell, Non-sinusoidal activity can produce crossfrequency coupling in cortical signals in the absence of functional interaction between neural sources, PLoS ONE 11, 1 (2016)

[57] A. B. Tort, R. Komorowski, H. Eichenbaum, and N. Kopell, Measuring phase-amplitude coupling between neuronal oscillations of different frequencies, Journal of Neurophysiology 104, 1195 (2010).

[58] M. C. Fellner, S. Gollwitzer, S. Rampp, G. Kreiselmeyr, D. Bush, B. Diehl, N. Axmacher, H. Hamer, and S. Hanslmayr, Spectral fingerprints or spectral tilt? Evidence for distinct oscillatory signatures of memory formation, bioRxiv , 1 (2018)

[59] J. Parvizi and S. Kastner, Promises and limitations of human intracranial electroencephalography, Nature Neuroscience 21, 474 (2018).

[60] G. Li, S. Jiang, S. E. Paraskevopoulou, M. Wang, Y. Xu, Z. Wu, L. Chen, D. Zhang, and G. Schalk, Optimal referencing for stereo-electroencephalographic (SEEG) recordings, NeuroImage 183, 327 (2018)

[61] M. R. Mercier, S. Bickel, P. Megevand, D. M. Groppe, C. E. Schroeder, A. D. Mehta, and F. A. Lado, Evaluation of cortical local field potential diffusion in stereotactic electro-encephalography recordings: A glimpse on white matter signal, NeuroImage 147, 219 (2017)

[62] E. Widjaja, M. Zamyadi, C. Raybaud, O. C. Snead, and M. L. Smith, Abnormal functional network connectivity among resting-state networks in children with frontal lobe epilepsy, American Journal of Neuroradiology 34, 2386 (2013).

[63] F. Pittau, C. Grova, F. Moeller, F. Dubeau, and J. Gotman, Patterns of altered functional connectivity in mesial temporal lobe epilepsy, Epilepsia 53, 1013 (2012)

[64] L. Caciagli, B. C. Bernhardt, S. J. Hong, A. Bernasconi, and N. Bernasconi, Functional network alterations and their structural substrate in drug-resistant epilepsy, Frontiers in Neuroscience 8, 1 (2014)

[65] B. Mišić, R. F. Betzel, A. Nematzadeh, J. Goñi, A. Griffa, P. Hagmann, A. Flammini, Y. Y. Ahn, and O. Sporns, Cooperative and Competitive Spreading Dynamics on the Human Connectome, Neuron 86, 1518 (2015)

[66] B. Vázquez-Rodríguez, Z.-Q. Liu, P. Hagmann, and B. Misic, Signal propagation via cortical hierarchies, Network Neuroscience , 1 (2020).

[67] S. M. Mitchell, S. Lange, and H. Brus, Gendered citation patterns in international relations journals, International Studies Perspectives 14, 485 (2013). 
[68] M. L. Dion, J. L. Sumner, and S. M. Mitchell, Gendered citation patterns across political science and social science methodology fields, Political Analysis 26, 312 (2018).

[69] N. Caplar, S. Tacchella, and S. Birrer, Quantitative evaluation of gender bias in astronomical publications from citation counts, Nature Astronomy 1, 0141 (2017).

[70] D. Maliniak, R. Powers, and B. F. Walter, The gender citation gap in international relations, International Organization 67, 889 (2013).
[71] J. D. Dworkin, K. A. Linn, E. G. Teich, P. Zurn, R. T. Shinohara, and D. S. Bassett, The extent and drivers of gender imbalance in neuroscience reference lists, bioRxiv 10.1101/2020.01.03.894378 (2020), https://www.biorxiv.org/content/early/2020/01/11/2020.01.03.8943

[72] D. Zhou, E. J. Cornblath, J. Stiso, E. G. Teich, J. D. Dworkin, A. S. Blevins, and D. S. Bassett, Gender diversity statement and code notebook v1.0 (2020). 